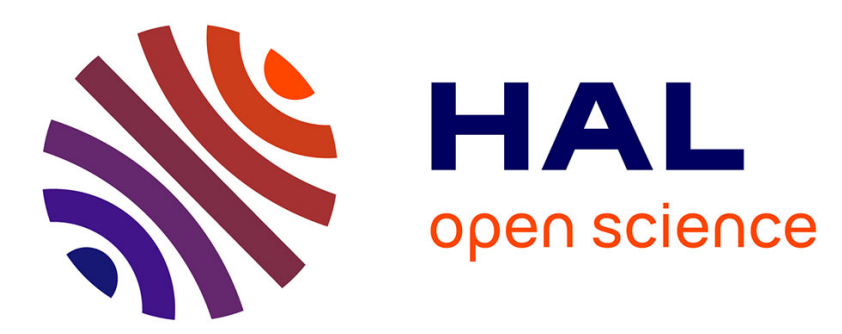

\title{
Effect of surface radiation on natural convective flows and onset of flow reversal in asymmetrically heated vertical channels
}

\author{
R. Li, M. Bousetta, Eric Chénier, Guy Lauriat
}

\section{To cite this version:}

R. Li, M. Bousetta, Eric Chénier, Guy Lauriat. Effect of surface radiation on natural convective flows and onset of flow reversal in asymmetrically heated vertical channels. International Journal of Thermal Sciences, 2013, 65 (-), pp.9-27. 10.1016/j.ijthermalsci.2012.10.023 . hal-00764760

\author{
HAL Id: hal-00764760 \\ https://hal.science/hal-00764760
}

Submitted on 13 Dec 2012

HAL is a multi-disciplinary open access archive for the deposit and dissemination of scientific research documents, whether they are published or not. The documents may come from teaching and research institutions in France or abroad, or from public or private research centers.
L'archive ouverte pluridisciplinaire HAL, est destinée au dépôt et à la diffusion de documents scientifiques de niveau recherche, publiés ou non, émanant des établissements d'enseignement et de recherche français ou étrangers, des laboratoires publics ou privés. 


\title{
Effect of surface radiation on natural convective flows and onset of flow reversal in asymmetrically heated vertical channels
}

\author{
R. Li, M. Bousetta, E. Chénier*, G. Lauriat \\ Laboratoire Modélisation et Simulation Multi Echelle, UMR 8208 CNRS, Université Paris-Est \\ Marne-la-Vallée, Bâtiment Lavoisier, 5 Boulevard Descartes, F-77454 MARNE-LA-VALLEE Cedex 2
}

\begin{abstract}
Numerical solutions on the influence of surface radiation on the laminar air flow induced by natural convection in vertical, asymmetrically-heated channels are discussed. Variable property effects are accounted for in a full-elliptic mathematical formulation. The density variation is determined from the state equation for ideal gas. The experimental design and data reported in Webb and Hill [1] are taken as the base cases for carrying out the computations. The occurrence of flow reversals is first considered and revisited for pure natural convection, and the Nusselt number correlations derived from the numerical results are favorably compared with those reported in [1]. It is shown that the general effect of surface radiation is to delete the onset of pocketlike recirculations at the top part of the channel, to reduce the heated wall temperatures, and to increase the facing wall temperatures. Comparisons with usual methods used for decoupling the surface radiation effects are discussed. In the range of parameters investigated, increases in differences between inlet and maximum wall temperatures up to $200 K$ are shown to have small influences on the flow field and negligible effects on heat transfer performances.
\end{abstract}

Keywords: Natural convection, surface radiation, vertical channels, flow reversal, variable property effects, numerical simulations

\section{Nomenclature}

$\begin{array}{ll}a & \text { thermal diffusivity }\left[\mathrm{m}^{2} / \mathrm{s}\right] \\ A & \text { aspect ratio, } A=2 H / D \\ B & \text { radiosity }\left[\mathrm{W} / \mathrm{m}^{2}\right] \\ c_{p} & \text { specific heat }[\mathrm{J} /(\mathrm{K} \cdot \mathrm{kg})] \\ d_{\psi} & \text { width of the recirculation }[\mathrm{m}], \text { Eqs. } 36 \text { and } 37 \\ D & \text { wall spacing }[\mathrm{m}]\end{array}$

\footnotetext{
${ }^{*}$ Corresponding author

Email address: Eric.Chenier@univ-paris-est.fr (E. Chénier)
} 
$R a$

$R a^{*}$

$t$

T

$u, w$

$$
\text { V }
$$$$
x, z
$$

\section{Greeks}

\section{Subscripts}

\section{H}

gravitational acceleration $\left[\mathrm{m} / \mathrm{s}^{2}\right]$

irradiation or incoming radiative heat flux density $\left[\mathrm{W} / \mathrm{m}^{2}\right]$

heat transfer coefficient $\left[W /\left(m^{2} \cdot K\right)\right]$

half of the total channel height $[m]$

thermal conductivity $[W /(m \cdot K)]$

kernel function (Eq. 10)

penetration length $[\mathrm{m}]$

mass flow rate $[\mathrm{kg} /(\mathrm{m} \cdot \mathrm{s})]$

molecular weight $[\mathrm{kg} / \mathrm{kmol}]$

mass flow rate in the entrance section, Eq. 34

mass flow rate leaving the channel, Eq. 35

conduction-to-radiation parameter, $N_{R}=q_{w} /\left(\sigma T_{0}^{4}\right)$

mean Nusselt number

numbers of grid points in $x$ - and $z$-directions

pressure $[\mathrm{Pa}]$

sum of static and hydrostatic pressures $[P a]$

pressure at the outlet section $[P a]$

dimensionless pressure, $p^{*}=p /\left(\rho_{0} w_{0}^{2}\right)$

Prandtl number, $\operatorname{Pr}=\nu_{0} / a_{0}$

heat flux density $\left[W / m^{2}\right]$

ideal gas constant, $R=8.3145[\mathrm{~J} /(\mathrm{mol} \cdot \mathrm{K})]$

modified Rayleigh number based on $q_{w}, R a=g \beta_{0} D^{3} q_{w} /\left(a_{0} \nu_{0} k_{0}\right)$

channel Rayleigh number, $R a^{*}=R a \times(D / H)$

time $[s]$

temperature $[K]$

velocity components $[\mathrm{m} / \mathrm{s}]$

velocity vector

coordinates $[m]$

order of consistency of the numerical scheme

thermal coefficient of volumetric expansion, $\beta=1 / T_{0}\left[K^{-1}\right]$

temperature difference scale, $\Delta T=q_{w} D / k_{0}[K]$

emissivity

non-Boussinesq parameter, $\gamma=\Delta T / T_{0}$

dynamic viscosity $[\mathrm{kg} /(\mathrm{m} \cdot \mathrm{s})]$

kinematic viscosity, $\nu=\mu / \rho\left[\mathrm{m}^{2} / \mathrm{s}\right]$

density $\left[\mathrm{kg} / \mathrm{m}^{3}\right]$

Stefan-Boltzmann constant, $\sigma=5.67 \cdot 10^{-8}\left[\mathrm{~W} /\left(\mathrm{m}^{2} \cdot \mathrm{K}^{4}\right)\right]$

dimensionless temperature, $\theta=\left(T-T_{0}\right) / \Delta T$

dimensionless time, $\tau=t \sqrt{g \beta_{0} \Delta T D} / D$

quantity based on channel height 


$\begin{array}{ll}c, i & \text { natural convection } \\ \max & \text { maximum } \\ r & \text { radiative } \\ w & \text { wall } \\ 0 & \text { inlet section } \\ t & \text { total }\end{array}$

\section{Superscripts}

$\begin{array}{ll}- & \text { averaged quantity } \\ * & \text { dimensionless quantity }\end{array}$

\section{Introduction}

Natural convection in vertical parallel-plate channel is relevant to a wide range of applications such as the cooling of electronic equipments in which circuit cards containing heat generating electronic devices are arrayed to form vertical channels, the design of solar panels, energy efficient buildings, heat removal in nuclear technology, and a host of others. The problem of natural convection in vertical channels has been the focus of extensive investigations since the pioneering work by Elenbass [2], resulting in numerous theoretical, numerical and experimental works. However, the number of studies reported on combined surface radiation and natural convection is very limited, despite radiative exchanges amongst surface plays a significant role for most practical applications.

Developing and fully developed laminar free convection between vertical flat plates with symmetric or asymmetric heating were reconsidered by Bodoia and Osterle [3], investigated analytically and experimentally by Aung [4], and Aung et al. [5]. These studies were the foundations for few others studies conducted later on. Amongst these, the experimental work by Wirtz and Stutzman [6] on developing natural convection of air flowing between vertical parallel plates with uniform and symmetric heat fluxes is frequently quoted. An up to date review on the formulas for the calculation of the heat transfer and flow rate for twodimensional natural convection between vertical plates having uniform wall temperature or uniform heat flux boundary conditions was presented by Olsson [7]. The recent experimental study conducted by Lu et al. [8] for channels with large aspect ratio and narrow gap, and with air as the working fluid, adds Nusselt number correlations for the case of symmetrical, uniformly heated vertical walls. Although high wall temperatures were measured (up to $\approx 600 \mathrm{~K}$ ), the radiation effects may be considered as weak owing to the symmetrical heating, small size of the apertures and large aspect ratios.

Recently, laminar natural convection of air in parallel-plate vertical microchannels was investigated theoretically and numerically in the transient regime, with velocity slip and temperature jump boundary conditions at the walls, by Buonomo and Manca [9, 10]. The 
microchannels were asymmetrically or symmetrically heated at uniform heat flux, and the boundary layer assumption was invoked for various values of the Knudsen number over the range of the first-order model for the continuum slip flow regime. Results showed overshoots in the transient maximum wall temperature. A composite correlation was proposed to estimate average Nusselt numbers at steady state.

\subsection{Flow reversals}

The theory of flow reversal of fully developed, aiding mixed convection and numerical solutions for developing flows were presented by Aung and Worku [11, 12]. Since these early studies, many numerical works dealing with the buoyancy effect on the flow structure and heat transfer have been performed (see for example [13-16]) while a limited number of experiments have been carried out.

The study of flow reversals in the case of pure natural convection in vertical channel was considered in only few works.

An experimental study for natural convection in a vertical channel with the heated wall maintained at uniform temperature and unheated facing wall was carried out with water as the working fluid by Sparrow et al. [17]. For the first time, the formation of pocket of recirculating flow at Rayleigh numbers exceeding a threshold value was revealed by flow visualizations. It was found that the recirculation, fed by fluid drawn into the outlet section of the channel adjacent to the adiabatic wall, has no effect on the heat transfer at the heated wall. As in the work by Bar-Cohen and Rohsenow [18], a new dimensionless group was introduced, the channel Rayleigh number $R a^{*}=R a_{D} \times(D / H)$, which has been shown to be the most convenient group for correlating the Nusselt number results. Since this pioneer work, the study of the flow reversals in natural convection has received only limited attention. The most salient studies are those by Kihm et al. [19] who identified the occurrence of the onset and penetration lengths of the flow reversal in natural convection of air through vertical isothermal channel walls, and the recent experimental study by Ospir et al. [20] on natural convection in an asymmetrically heated vertical plane channel with water as the working fluid. Flow visualization techniques based on laser tomography were used in [20] for investigating the flow structure in steady-state, boundary layer flow regime as well as in the early stage following the beginning of the heating. From the experiments carried out for several values of the modified Rayleigh number and channel aspect ratios, very new insights on the reversed flow structures were revealed.

\subsection{Coupled natural convection and radiation}

The problem of combined radiation and natural convection was extensively studied, specially for closed cavities. It can be classified into two categories: one only with wall radiations, and the other with both wall and gas radiations. For cases including gas radiation combined with natural convection heat transfer, which is always important for combustion and engineering applications, numerous papers have been published. Yamada et al. [21], Colomer et al. [22] 
and, more recently, Mondal and Li [23], who used an improved lattice-Boltzmann method, presented relevant literature surveys on this subject. The present study encompasses only combined surface radiation and natural convection that arises when the working fluid is assumed transparent to infrared radiations.

\subsubsection{Coupled natural convection and surface radiation in cavities}

Numerical investigation of combined surface radiation and natural convection in a vertical square, differentially heated cavity were reported by Balaji and Venkateshan [24]. They showed that radiation has a dual effect of contributing to the overall heat transfer as well as decreasing the convective component. They underlined that simple formulae that account for radiation in an additive way are not adequate. In a subsequent study, Balaji and Venkateshan [25] proposed correlations for both convective and radiative heat transfers for air as the working fluid. These correlations were found in fair agreement with the results from an experimental study conducted by Ramesh and Venkateshan [26].

The impact of surface radiation on multiple solutions inside a square two-dimensional cavity heated from below was studied numerically by Ridouane et al. [27]. The emissivities of the isothermal horizontal walls were different from those of the vertical adiabatic walls and were set to $\epsilon=0.05$ or to $\epsilon=0.85$. Four combinations of wall emissivities were considered. In comparison with pure natural convection, it was shown that the ranges of the steady-state modes as well as the nature and magnitude of periodic solutions were highly affected by surface radiation.

This problem was reconsidered more recently by Gad and Balaji [28] for air-filled, rectangular cavities heated from below with surface radiation spanning six different aspect ratios (from $A=1$ to $A=10$ ). The critical Rayleigh number for the onset of convection was determined as function of two parameters: the emissivity of the adiabatic sidewalls and the aspect ratio for a cold top wall at $T_{c}=303 \mathrm{~K}$, the emissivity of the horizontal walls being set to $\varepsilon=0.85$. It was shown that the onset of Rayleigh-Bénard convection is delayed with an increase in the emissivity of the sidewalls and, that the effect of surface radiation diminishes with an increase in the aspect ratio.

Natural convection for air in an open-ended cavity heated from above was experimentally studied by Manca and Nardini [29] in the case of high emissivity of the horizontal walls. They observed that surface radiation caused a temperature increase in the unheated lower plate, which could rise to secondary motions due to plumes inside the cavity. Correlations for average Nusselt numbers and maximum wall temperatures were proposed.

Studies on combined natural convection and surface radiation from a heated body inside a cavity are more scarse (for an overview of recent references, see Bouali et al. [30], Lauriat and Desrayaud [31], Mezrhab et al. [32]). In the paper by Mezrhab et al. [32], a differentiallyheated cavity of square cross-section containing a conducting, centered square body was 
numerically investigated. Amongst the conclusions drawn for this particular configuration, the effect of the inner body on the flow field and heat transfer was shown much important when surface radiation exchanges were taken into account. In the range of Rayleigh number considered, it was also shown that radiation augments the fluid velocities in comparison with those obtained for a cavity without an inner body. However, much of these conclusions are specific to the geometrical case considered, as underlined in Sun et al. [33].

\subsubsection{Coupled natural convection and radiation in vertical parallel plate channels}

The interaction of surface radiation with developing laminar natural convection in vertical parallel plate channels with asymmetric heating was numerically studied by Carpenter et al. [34]. A parabolic formulation of the governing equations was employed and the Boussinesq approximation invoked. They investigated the effects of the five dimensionless parameters involved in the dimensionless formulation (heat flux ratio, Rayleigh number, aspect ratio, emissivity, and radiation number), and showed that radiation significantly alters the pure natural convection results by reducing the wall temperatures, especially the maximum value.

The effect of surface radiation was reconsidered by Webb and Hill [1] in their paper reporting on experiments designed to determine local and average heat transfer characteristics for natural convection in a vertical parallel plate channel, one wall heated with uniform heat flux and the other thermally insulated. Local temperatures along both walls were collected for a wide range of heating rates and wall spacings corresponding to the high channel Rayleigh number regime (i.e. $503 \leq R a^{*} \leq 1.75 \cdot 10^{7}$ ). Unheated entry and exit lengths $(7.62 \mathrm{~cm} \approx H / 2)$ were added to the heated section $(H=15.2 \mathrm{~cm})$ to minimize radiation losses near the channel entrance and exit. The local radiative heat flux was determined from the temperature data by solving the system of equations describing the radiation exchanges for channel walls assumed gray and diffuse with an emissivity measured to $\varepsilon=0.1$. The heating rate was then reduced by the calculated local radiation loss, and the corrected heat transfer results were used in all correlations presented in the paper. The results compared favorably with previous experimental data, and the importance of corrections for radiation and conduction losses as well as the use of local thermophysical properties in correlating the data were underlined.

The experimental design, wall spacings and heating rates described in [1] were numerically simulated, as accurately as possible, in the preparation of the present paper.

Combined natural convection and thermal radiation in vertical parallel plate channels was experimentally investigated by Manca and Naso [35]. The measurements showed that the effect of surface radiation is more important for asymmetric heating than for symmetric heating. In addition, it was emphasized that the flow patterns tend to those of symmetric heating for high wall emissivities, and reduce the Rayleigh number corresponding to the developing regime. Correlations between local Nusselt numbers at various emissivities have also been proposed. 
Laminar natural convection and surface radiation between vertical parallel plates, a central, highly emissive $(\varepsilon=0.85)$ hot plate and two unheated polished plates $(\varepsilon=0.05)$, was investigated experimentally by Krishnan et al. [36] for various plate spacings. The temperature measurements were introduced as boundary conditions into a numerical code in order to obtain the convective heat transfer rates. This study brought out the significance of radiation at room temperature. A correlation for the average convective wall heat transfer was derived.

\subsection{Channel extensions}

Amongst the attempts for solving natural convection at low channel Rayleigh number, is the use of channel extensions as it was suggested in many previous numerical studies that we briefly review in what follows.

Naylor et al. [37] solved the full elliptic forms of the governing equations for pure natural convection using inlet flow boundary conditions based on the Jeffrey-Hamel flow in order to represent more realistically the entrance flow. Their solutions validated the inlet pressure approximation $\left(p=-\rho_{0} w^{2} / 2\right)$ commonly used in parabolic formulations. Their predictions of fluid separation at the channel inlet is not in agreement with most of the elliptic solutions published so far, and appear to be caused by the shape of the extension. The optimization of plate separation of an open, vertical, parallel-plate channel that is cooled by natural convection of air with the plates symmetrically heated by uniform heat flux has been studied by Morrone et al. [38] by solving the full elliptic conservation equations in a I-shaped computational domain. Correlations for the dimensionless flow rate and optimal values of the spacing were derived and compared with the predictions of Bar-Cohen and Rohsenow [18] and Anand et al. [39].

Natural convection of air in channel-chimney systems was studied experimentally [40], and numerically by using the stream function-vorticity approach and the control volume method by Andreozzi et al. [41, 42] for vertical channels heated symmetrically at uniform heat flux and with adiabatic extensions. Results obtained provide guidelines to determine if the channel-chimney system is in critical condition related to flow reattachment or separation.

Closely related to these approaches is the paper by Bello-Ochende and Bejan [43]: the channel was fitted with upstream and downstream extensions whose lengths were selected based on accuracy tests. For the downstream extension domain, free slip and no penetration were specified at one side and zero stress at the other side in order to nullify the chimney effect. From our point of view, this procedure leads to unrealistic outflow fields for a periodic array of vertical channels.

As shown in a recent paper by Sun et al. [44], the use of inlet channel extensions is not required, even for rather low channel Rayleigh numbers, since a regularization procedure 
allows to numerically solve the problem with a good accuracy. On the other hand, extensions are required for the very low Rayleigh numbers at which backward thermal diffusion dominates. Upper extensions lead obviously to other problems owing to superimposition of chimney effects.

\subsection{Aim of the present study}

This paper reports a numerical study of a combined radiation and natural convection problem between vertical plates with asymmetric heating. The present study differs from the aforementioned ones in the following issues:

- the problem formulation is based on a weakly compressible formulation in order to account for unpredictable maximum temperature difference for uniformly heated walls. Experimental studies reveal indeed surface-to-ambient temperature difference up to about $500 \mathrm{~K}$ according to the heating rate and/or wall spacings when air is the working fluid $[8,45]$. The dynamical inlet/outlet boundary conditions differ also;

- the flow reversal phenomenon for pure natural convection is revisited;

- the effects of surface radiation as well on the flow structure as on the heat transfer rate are investigated and supported through comparisons with the experimental study by Webb and Hill [1].

\section{Problem statement}

\subsection{Dimensional form}

The fluid flow and heat transfer are governed by the Navier-Stokes and energy equations for a two-dimensional, laminar flow of a Newtonian fluid. The viscous dissipation term in the energy equation is neglected and the fluid is assumed transparent to thermal radiation leaving the channel walls. The energy equation is coupled with the equation dealing with radiant interchanges amongst surfaces through the thermal boundary conditions. Since some calculations were carried out for maximum temperature differences larger than that possible for an accurate use of the Boussinesq approximation for ideal gases $\left(\Delta T_{\max } \leq 0.1 T_{0}\right.$, where $T_{0}$ is a reference temperature), a weakly compressible formulation was employed by assuming the pressure work in the energy equation as negligible and, by calculating the density field from the state equation with a uniform thermodynamic pressure within the channel. The conservation equations are written as follows:

$$
\begin{gathered}
\frac{\partial \rho}{\partial t}+\nabla \cdot(\rho \mathbf{v})=0 \\
\frac{\partial(\rho \mathbf{v})}{\partial t}+\nabla \cdot(\rho \mathbf{v} \otimes \mathbf{v})=-\nabla\left(p+\rho_{0} g z\right)+\nabla \cdot\left[\mu\left(\nabla \mathbf{v}+\nabla^{t} \mathbf{v}-\frac{2}{3} \nabla \cdot \mathbf{v}\right)\right]+\left(\rho_{0}-\rho\right) g \mathbf{e}_{z}
\end{gathered}
$$




$$
c_{p}\left(\frac{\partial(\rho T)}{\partial t}+\nabla \cdot(\rho \mathbf{v} T)\right)=\nabla \cdot(k \nabla T)
$$

where $\rho_{0}$ in the momentum equation is the density at a reference temperature, chosen as the inlet fluid temperature and $\mathbf{e}_{z}$ is the unit vector along the $z$-axis, pointing upward. All thermophysical properties are considered as temperature dependent, i.e. $\rho(T), \mu(T), c_{p}(T)$ and $k(T)$. For dry air, $c_{p}(T), k(T)$ and $\mu(T)$ are calculated according to the polynomial relationships given by Lide and Kehiaian [46], valid in the range [100 K, $600 \mathrm{~K}$ ]. These properties variations were used in [47] where the specific relationships employed herein can be found.

The system of conservation equations is completed by the ideal gas law used for the calculations of the density at any point $\mathbf{M}$ :

$$
\rho(\mathbf{M}, t)=\frac{\bar{P}_{0} M_{a}}{R T(\mathbf{M}, t)}
$$

$\bar{P}_{0}$ is the uniform thermodynamic pressure, equal the average hydrostatic pressure in the surroundings, $\bar{P}_{0}=P(0)-\rho g H$.

\section{Boundary conditions:}

- at the vertical walls:

$$
-x=0, \quad 0<z<H / 2 \text { and } 3 H / 2<z<2 H,
$$

$$
\mathbf{v}=\mathbf{0}, \quad q_{r}(z)-k \frac{\partial T}{\partial x}=0
$$

where $q_{r}$ is the net radiative heat flux density along the surface.

- $x=0$ and $H / 2<z<3 H / 2$,

$$
\mathbf{v}=\mathbf{0}, \quad q_{r}(z)-k \frac{\partial T}{\partial x}=q_{w}
$$

$-x=D$ and $0<z<2 H$,

$$
\mathbf{v}=\mathbf{0}, \quad q_{r}(z)+k \frac{\partial T}{\partial x}=0
$$

- at the inlet/outlet sections:

$-0<x<D$ and $z=0$ or $z=2 H$,

$$
u=0, \frac{\partial w}{\partial z}=0, \quad\left\{\begin{array}{l}
\text { if } \mathbf{v} \cdot \mathbf{n}<0 \text { (inlet condition), } T=T_{0}, p_{g}=-\rho \frac{w^{2}}{2} \\
\text { if } \mathbf{v} \cdot \mathbf{n} \geq 0 \text { (outlet condition), } \frac{\partial T}{\partial z}=0, p_{g}=0
\end{array}\right.
$$


where $p_{g}=p+\rho_{0} g z$ is the departure of the pressure from the hydrostatic pressure and $\mathbf{n}$ is the unit normal vector pointing outward the computational domain. The inlet/outlet conditions (Eq. 8) for the velocity assume that the fluid enters or exits normally to the inlet/outlet sections.

The pressure and temperature boundary conditions at the bottom and top sections depend on whether the fluid enters or exits the channel:

* In the first case $(\mathbf{v} \cdot \mathbf{n}<0)$, the temperature equals the surroundings temperature and the static and hydrostatic pressure drops equilibrate the increase in kinetic energy for non-viscous fluids.

$\star$ In the second case $(\mathbf{v} \cdot \mathbf{n} \geq 0)$, the heat diffusion normal to the inlet/outlet section is neglected and the fluid flow is assumed to behave as a jet with parallel streamlines. With the jet approximation, the sum of the static and hydrostatic pressures is kept constant at the outlet section and equal to its surrounding value which is arbitrary fixed to zero.

\subsubsection{Surface-to-surface radiation model}

The radiative exchanges amongst the surfaces were calculated by considering the channel as a two-dimensional enclosure consisting of four gray-diffuse, vertical surfaces (the three parts of the left-hand side wall and the adiabatic right-hand side wall) and two horizontal surfaces regarded as black radiators at an effective temperature $T_{0}$. Since the vertical walls have the same emissivity, $\varepsilon$, the temperature distributions are evaluated from

$$
\sigma T_{i}^{4}\left(\mathbf{r}_{i}\right)=\frac{1-\varepsilon}{\varepsilon} q_{i}+B_{i}\left(\mathbf{r}_{i}\right)
$$

The position vector $\mathbf{r}_{i}$ denotes the locations of elementary surfaces $d S_{i}$ on surface $S_{i}$, and $B_{i}\left(\mathbf{r}_{i}\right)$ is the radiosity, which is the radiation heat flux density leaving surface $S_{i}$. The radiosity distributions along the vertical walls are defined as

$$
B_{i}\left(\mathbf{r}_{i}\right)=q_{i}\left(\mathbf{r}_{i}\right)+\sum_{j=1}^{6} \int_{S_{j}} B_{j}\left(\mathbf{r}_{j}\right) K\left(\mathbf{r}_{i}, \mathbf{r}_{j}\right) d S_{j}=q_{i}\left(\mathbf{r}_{i}\right)+G_{i}\left(\mathbf{r}_{i}\right)
$$

The kernel function $K$ reads $K\left(\mathbf{r}_{i}, \mathbf{r}_{j}\right)=\cos \left(\theta_{i}\right) \cos \left(\theta_{j}\right) / \pi r_{i j}^{2}$ [48]. For plane or convex surfaces, $K\left(\mathbf{r}_{i}, \mathbf{r}_{i}\right)=0$. The term $G_{i}\left(\mathbf{r}_{i}\right)$ is the radiative heat flux density incident on surface $S_{i}$. At the channel apertures, $B_{i}\left(\mathbf{r}_{i}\right)=\sigma T_{0}^{4}$.

The preceding set of equations may be combined to yield a system of integral equations that relates temperatures and heat fluxes at the six surfaces. There follows

$$
\sigma T_{i}^{4}\left(\mathbf{r}_{i}\right)-\frac{q_{i}\left(\mathbf{r}_{i}\right)}{\varepsilon_{i}}=\sum_{j=1}^{6} \int_{S_{j}} \sigma T_{j}^{4}\left(\mathbf{r}_{j}\right) K\left(\mathbf{r}_{i}, \mathbf{r}_{j}\right) d S_{j}-\sum_{j=1}^{6} \int_{S_{j}} \frac{1-\varepsilon_{j}}{\varepsilon_{j}} q_{j}\left(\mathbf{r}_{j}\right) K\left(\mathbf{r}_{i}, \mathbf{r}_{j}\right) d S_{j} \quad 1 \leq i \leq 6
$$


The above system of six integral equations contains six unknown functions: the four distributions of temperature along the vertical walls and the two heat flux distributions at the channel apertures.

\subsubsection{Wall heat fluxes and heat transfer coefficients}

For a uniform heat flux density $q_{w}$ prescribed at the heated surface, the local convective, $q_{c}(z)$ and radiative, $q_{r}(z)$ fluxes must balance $q_{w}$. Therefore

$$
q_{w}=q_{c}(z)+q_{r}(z)=k(z) \frac{\partial T_{w}(z)}{\partial x} \mathbf{n} \cdot \mathbf{e}_{x}+B(z)-G(z)
$$

where $G(z)$ is the local irradiation onto the heated surface (second right-hand side term in Eq. 10). The local convective heat transfer coefficient is determined using the relation (refer to [1]):

$$
h(z)=\frac{q_{w}-q_{r}(z)}{T_{w}(z)-T_{0}}
$$

while a local radiative heat transfer coefficient may be defined as

$$
h_{r}(z)=\frac{q_{r}(z)}{T_{w}(z)-T_{0}}
$$

The total heat transfer coefficient is thus $h_{t}(z)=h(z)+h_{r}(z)$. Average heat transfer coefficients over the heated surface $(x=0, H / 2 \leq z \leq 3 H / 2)$ are calculated according to the relations

$$
\bar{h}_{c, 1}=\frac{1}{H} \int_{\frac{H}{2}}^{\frac{3 H}{2}} h(z) d z \quad \text { and } \quad \bar{h}_{r, 1}=\frac{1}{H} \int_{\frac{H}{2}}^{\frac{3 H}{2}} h_{r}(z) d z
$$

and $\bar{h}_{t, 1}=\bar{h}_{c, 1}+\bar{h}_{r, 1}$.

The heat flux conservation at the heated surface may also be stated as:

$$
q_{w}=\bar{q}_{c}+\bar{q}_{r}
$$

Average heat fluxes are calculated by averaging $q_{c}(z)$ and $q_{r}(z)$. This leads to

$$
\bar{q}_{c}=\frac{1}{H} \int_{\frac{H}{2}}^{\frac{3 H}{2}} k(z) \frac{\partial T_{w}(z)}{\partial x} \mathbf{n} \cdot \mathbf{e}_{x} d z \quad \text { and } \quad \bar{q}_{r}=\frac{1}{H} \int_{\frac{H}{2}}^{\frac{3 H}{2}}(B(z)-G(z)) d z
$$

It should be noted that two different average heat transfer coefficients may be also determined using the two following relations (refer to Bianco et al. [49]):

$$
\bar{h}_{c, 2}=\frac{\bar{q}_{c}}{\bar{T}_{w}-T_{0}} \quad \text { and } \quad \bar{h}_{r, 2}=\frac{\bar{q}_{r}}{\bar{T}_{w}-T_{0}}
$$

where $\bar{T}_{w}$ is the average temperature of the heated wall. The main advantage in using the above definitions is that a heat transfer correlation yields automatically $\bar{T}_{w}$ when the input 
is the wall heat flux.

As a result, if the wall temperatures are recorded from experiments, as in $[1,36]$, the radiative and total heat transfer coefficient can be calculated accurately, and the convective heat transfer coefficient can be deduced by using either Eq. 15 or Eq. 18. However, this convective heat transfer is meaningful in pure convection only if the surface radiation exchanges modify slightly the temperature distribution along the heated wall. This point will be examined in what follows.

\subsection{Dimensionless form}

\subsubsection{Fluid flow and surface-to-surface radiation equations}

The governing equations are cast in dimensionless form using the reference length scale $D$, time scale $D /\left(g \beta_{0} \Delta T D\right)^{0.5}$ where $\beta_{0}=1 / T_{0}$, the velocity scale $\left(g \beta_{0} \Delta T D\right)^{0.5}$, the dynamic pressure scale $\rho_{0} g \beta_{0} \Delta T D$, the temperature difference scale $\Delta T=q_{w} D / k_{0}$, and the radiative heat flux scale $\sigma T_{0}^{4}$. Note that these scales are those often used when invoking the Boussinesq approximation for boundary-layer type flows. The resulting set of governing equations reads:

$$
\begin{gathered}
\frac{\partial \rho^{*}}{\partial \tau}+\nabla \cdot\left(\rho^{*} \mathbf{v}^{*}\right)=0 \\
\frac{\partial\left(\rho^{*} \mathbf{v}^{*}\right)}{\partial \tau}+\nabla \cdot\left(\rho^{*} \mathbf{v}^{*} \otimes \mathbf{v}^{*}\right)=-\nabla\left(p^{*}+\frac{1}{\gamma} z^{*}\right)+\sqrt{\frac{P r}{R a}} \nabla \cdot\left[\mu^{*}\left(\nabla \mathbf{v}^{*}+\nabla^{t} \mathbf{v}^{*}-\frac{2}{3} \nabla \cdot \mathbf{v}^{*}\right)\right]+\frac{1}{\gamma}\left(1-\rho^{*}\right) \mathbf{e}_{z} \\
c_{p}^{*}\left(\frac{\partial\left(\rho^{*} \theta\right)}{\partial \tau}+\nabla \cdot\left(\rho^{*} \mathbf{v}^{*} \theta\right)\right)=\frac{1}{\sqrt{R a P r}} \nabla \cdot\left(k^{*} \nabla \theta\right) \\
\rho^{*}=\frac{1}{(\gamma \theta+1)}
\end{gathered}
$$

In the above equations, superscript * indicates dimensionless dependent quantities and dimensionless fluid properties with the reference properties being those at $T_{0}$. The dimensionless temperature difference is $\theta=\left(T-T_{0}\right) / \Delta T$. The three dimensionless parameters are the Prandtl and Rayleigh numbers and, the non-Boussinesq parameter, $\gamma=\Delta T / T_{0}$, that characterizes the departure from the Boussinesq approximation. The dimensionless form of Eq. 11 writes

$$
T_{i}^{*^{4}}\left(\mathbf{r}_{i}^{*}\right)-\frac{q_{i}^{*}\left(\mathbf{r}_{i}^{*}\right)}{\varepsilon_{i}}=\sum_{j=1}^{6} \int_{S_{j}^{*}} T_{j}^{*^{4}}\left(\mathbf{r}_{j}^{*}\right) K\left(\mathbf{r}_{i}^{*}, \mathbf{r}_{j}^{*}\right) d S_{j}^{*}-\sum_{j=1}^{6} \int_{S_{j}^{*}} \frac{1-\varepsilon_{j}}{\varepsilon_{j}} q_{j}^{*}\left(\mathbf{r}_{j}^{*}\right) K\left(\mathbf{r}_{i}^{*}, \mathbf{r}_{j}^{*}\right) d S_{j}^{*} \quad 1 \leq i \leq 6
$$

where $T^{*}=\gamma \theta+1$ and $q_{r, i}^{*}=q_{r, i} / \sigma T_{0}^{4}$. 


\subsubsection{Boundary conditions}

- at the vertical walls:

$-x^{*}=0, \quad 0<z^{*}<A / 4$ and $3 A / 4<z^{*}<A$,

$$
\mathbf{v}^{*}=\mathbf{0}, \quad q_{r}^{*}\left(z^{*}\right)-N_{R} k^{*} \frac{\partial \theta}{\partial x^{*}}=0
$$

- $x^{*}=0$ and $A / 4<z^{*}<3 A / 4$,

$$
\mathbf{v}^{*}=\mathbf{0}, \quad q_{r}^{*}\left(z^{*}\right)-N_{R} k^{*} \frac{\partial \theta}{\partial x^{*}}=N_{R}
$$

$-x^{*}=1$ and $0<z^{*}<A$,

$$
\mathbf{v}^{*}=\mathbf{0}, \quad q_{r}^{*}\left(z^{*}\right)+N_{R} k^{*} \frac{\partial \theta}{\partial x^{*}}=0
$$

- at the inlet/outlet sections:

$$
\begin{aligned}
& -0<x^{*}<1 \text { and } z^{*}=0 \text { or } z^{*}=A, \\
& u^{*}=0, \frac{\partial w^{*}}{\partial z^{*}}=0,\left\{\begin{array}{l}
\text { if } \mathbf{v}^{*} \cdot \mathbf{n}<0 \text { (inlet condition), } \theta=0, p_{g}^{*}=-\frac{w^{* 2}}{2} \\
\text { if } \mathbf{v}^{*} \cdot \mathbf{n} \geq 0 \text { (outlet condition), } \frac{\partial \theta}{\partial z^{*}}=0, p_{g}^{*}=0
\end{array}\right.
\end{aligned}
$$

The additional dimensionless parameters involved in the boundary conditions are the channel aspect ratio, $A=2 H / D$, and $N_{R}=q_{w} / \sigma T_{0}^{4}$, usually called the conduction-to-radiation parameter for the effects of surface radiation without linearization of the radiative heat flux [48].

Finally, the above mathematical modeling shows that the problem of two-dimensional, laminar natural convection of a gas through a channel subjected to a prescribed heat flux at one section of the walls while the others are assumed adiabatic (or subjected to the same heat flux density), involves six dimensionless parameters (not accounted for is the reference temperature $T_{0}$ introduced in the relations used for modeling the temperature dependence of the thermophysical properties): $A, \operatorname{Pr}, R a, \gamma, N_{R}$ and $\varepsilon$. The last three parameters account for variable properties and surface radiation effects.

Owing to this number of parameters, the derivation of a general Nusselt number correlation appears to be rather cumbersome. Note that the number of parameters is still 6 even if the maximum possible temperature difference is compatible with the validity of the Boussinesq approximation. This approximation makes sense a priori only if the maximum temperature difference is prescribed. A large class of practical applications, such as the cooling of electronic or lighting equipments (HB-LED, for example) does not match this requirement. 


\subsubsection{Correlations for average channel Nusselt numbers and maximum wall temperature}

For pure natural convection, the flow and heat transfer are governed by the height to width ratio $H / D$, the Rayleigh number, the Prandtl number, and the non-Boussinesq parameter. For an asymmetric heating the following Rayleigh numbers are used:

$$
R a=\frac{g \beta_{0} q_{w} D^{4}}{a_{0} \nu_{0} k_{0}} \text { and } R a^{*}=R a \times \frac{D}{H} \text { the modified or channel Rayleigh number }
$$

As in many of the previous studies $[1,8,50]$, we did not use local Rayleigh numbers in what follows for deriving Nusselt number or $\theta_{\max }$ correlations. Correlation equations were determined for the local Nusselt number at midheight of the heated plate, for average Nusselt numbers based either on Eq. 15 or 18, and for maximal heated wall temperature:

$$
N u_{\frac{1}{2}}=\frac{q_{w} D}{2 k_{0}\left(T_{\frac{1}{2}}-T_{0}\right)}=a^{*} R a^{*^{m}} \quad, \overline{N u}=b^{*} R a^{*^{n}} \quad \text { and } \quad \theta_{\max }=c^{*} R a^{*^{-p}}
$$

with $T_{\frac{1}{2}}$ the average of the two wall temperature at $z=H$ and $\theta_{\max }=k_{0}\left(T_{\max }-T_{0}\right) / q_{w} D$; the definition of $N u_{\frac{1}{2}}$ refers to $[1,5]$. The convective, radiative and total average Nusselt number are calculated according to the relations

$\overline{N u}_{c, i}=\frac{\bar{h}_{c, i} D}{k_{0}}, \overline{N u}_{r, i}=\frac{\bar{h}_{r, i} D}{k_{0}}, \overline{N u}_{t, i}=\overline{N u}_{c, i}+\overline{N u}_{r, i} \quad$ for $\quad i=1,2$ (see Eqs. 15 and 18)

It should be noted that the fluid thermal conductivity is that at the inlet temperature because, from a practical viewpoint, the only available data in the design of a uniformly heated, flat plate channel are the wall heat flux, the inlet temperature and the dimensions of the channel.

\section{Numerical methods}

\subsection{Numerical scheme}

The equations for mass (Eq. 1), momentum (Eq. 2) and energy (Eq. 3) are discretized by a collocated finite volume scheme based on methods initially developed for incompressible fluid flows on unstructured meshes and for fully coupled velocity-pressure equations [51], and later on extended to the low Mach number approximation [52]. This latter method has been adapted to a velocity-pressure decoupling scheme and applied here for structured rectangular grids. Details on the numerical method can be found in [53].

Some key points of the spatial discretization are briefly recalled. Variables are located at the center of the rectangular cells. The thermal diffusion and the viscous part $\nabla \cdot(\mu \nabla \mathbf{v})$ are discretized with the classical 5 points-scheme with fluxes evaluated on cell faces with a twopoints approximation. In order to mimic as much as possible the kinetic energy balance (and the quadratic form of the thermal balance), additional conditions must be fulfilled. First, 
the discrete expression of the pressure gradient is the dual form of the discrete divergence of the velocity. Then, the mass flow rate acting in the transport contribution is the same as the one used in mass equation. Moreover, the convected quantities (the velocity components and the temperature) are evaluated on cell faces with a centered approximation, even if the mesh is irregular. Finally, the remaining viscous contributions $\nabla \cdot\left(\mu(\nabla \mathbf{v})^{t}-2 / 3 \mu(\nabla \cdot \mathbf{v}) \overline{\bar{I}}\right)$, with $\overline{\bar{I}}$ the unit tensor, are expressed with a weak formulation. It is worth noticing that mimicking the energy balances is also a convenient way to ensure some numerical stability of the numerical solutions provided the scheme is fully implicit.

A second order implicit Euler scheme is used for diffusion, with Adams-Bashforth extrapolations in time for convective and advective terms, and for the viscous part $\nabla$. $\left(\mu(\nabla \mathbf{v})^{t}-2 / 3 \mu(\nabla \cdot \mathbf{v}) \overline{\bar{I}}\right)$. The velocity-pressure decoupling is provided by a projection method [54]. The pressure correction $\Phi^{n+1}=p^{n+1}-p^{n}$ is solution of the Poisson problem:

$$
\nabla^{2} \Phi^{n+1}=f
$$

where $f$ is an appropriate source term and $\nabla^{2} \Phi^{n+1} \equiv \nabla \cdot\left(\nabla \Phi^{n+1}\right)$, with $\nabla \cdot($.$) and \nabla($. the collocated divergence and gradient. The solution of such a problem is well known to produce unrealistic checkerboard oscillations in the solution. To prevent this, the collocated Laplacian $\nabla^{2}($.$) in (Eq. 31) is substituted by the stable 5-points Laplacian \widetilde{\nabla}^{2}($.$) defined on$ staggered grids:

$$
\widetilde{\nabla}^{2} \Phi^{n+1}=f
$$

The view factors between the elements of the discretized wall surfaces and of the inlet/outlet sections (facets) were calculated by Hottel's crossed-string method. The coupling between the discretized energy equation and radiosity equation was first handled by discretization of the integral term in Eq. 10 by using the 1-point Gauss method, leading to a linear system of equations. Then, starting from the known wall temperatures at time $t^{n}$, we calculate the radiative heat fluxes on each facet. The radiative heat fluxes are then introduced into the boundary conditions (Eq. 5-7) to get new temperatures at the time $t^{n+1}$.

The resolution of the linear system of radiosity equations was performed by the Gauss method with partial pivoting. Solutions of Helmholtz problems for both the components of velocity and the temperature field, and the solution of the pressure correction equation (Eq. 32) were obtained by the Bi-Conjugate Gradient Stabilized method, preconditioned by an incomplete LU-decomposition. The time step value was controlled by fixing the upper bound of the Courant-Friedrich-Levy number, for example $C F L=0.5$. The stationary solution was assumed reached when $E^{n, n+1}<10^{-6}$ with

$$
E^{n, n+1}=\max \left(\frac{\left\|u^{\mathrm{n}+1}-u^{\mathrm{n}}\right\|_{2}}{\left\|u^{\mathrm{n}+1}\right\|_{2} \Delta \mathrm{t}^{\mathrm{n}}}, \frac{\left\|w^{\mathrm{n}+1}-w^{\mathrm{n}}\right\|_{2}}{\left\|w^{\mathrm{n}+1}\right\|_{2} \Delta \mathrm{t}^{\mathrm{n}}}, \frac{\left\|T^{\mathrm{n}+1}-T^{\mathrm{n}}\right\|_{2}}{\left\|T^{\mathrm{n}+1}\right\|_{2} \Delta \mathrm{t}^{\mathrm{n}}}\right)
$$

where $\|\cdot\|_{2}$ is the discrete norm $L_{2}$ and $\Delta t^{n}=t^{n+1}-t^{n}$. 


\subsection{Validation}

A thorough check of the dependence of numerical solutions with the mesh size was conducted for the natural convection case, with $H=15.2 \mathrm{~cm}$ and two plate spacings, $D=7.1 \mathrm{~cm}$ and $D=1.71 \mathrm{~cm}$ (Tab. 1). The mesh consists of $n_{x} \times n_{z}$ cells uniformly distributed in the vertical direction. To accurately represent the dynamic and thermal boundary layers, the grid was refined in the horizontal direction with a ratio between the largest and smallest cells equal to 5 . Table 1 provides:

- the mass flow rate in the entrance section,

$$
\dot{m}_{i n}=\int_{0}^{D} \rho(x, 0) w(x, 0) d x
$$

the mass flow rate leaving the channel (only the positive values of the vertical velocity are taken into account)

$$
\dot{m}_{\text {out }}=\int_{0}^{D} \rho(x, 2 H) \frac{w(x, 2 H)+|w(x, 2 H)|}{2} d x
$$

- the widths of the recirculation at the outlet of the channel $(z=2 H)$ and in the section of the outlet of the heated wall $(z=3 H / 2)$ are calculated from

$$
\begin{gathered}
d_{\psi}(2 H)=D-x_{1} \text { with } \psi\left(x_{1}, 2 H\right)=\psi_{w} \\
d_{\psi}(3 H / 2)=D-x_{2} \text { with } \psi\left(x_{2}, 3 H / 2\right)=\psi_{w}
\end{gathered}
$$

with $\psi_{w}$ the value of the stream function along the right plate, arbitrary set to zero;

- the two convective coefficients $\bar{h}_{c, 1}$ and $\bar{h}_{c, 2}$.

Based on data reported in Tab. 1, we adopted the mesh with the smallest grid size to simulate the natural convection flows, i.e. the mesh $160 \times 3200$. On the other hand, since the coupled problems are much more costly in computational time, the slightly coarser grid $80 \times 1600$ is chosen for computing fluid flows and heat transfers when surface-to-surface radiations are accounted for.

Some comparisons are also made with the results reported in [16] for mixed convection of air $(\operatorname{Pr}=0.71)$. In that configuration, the aspect ratio of the whole vertical channel is $A=12$, and flow reversal occurs for some set of Reynolds number, Re, and Richardson number $R i=R a /\left(\operatorname{Pr} R e^{2}\right)$. The entire left wall is subjected to a heat flux whereas the opposite plate is thermally isolated. The solid boundaries are perfectly reflecting surfaces $(\varepsilon=0)$. The inlet conditions (Eq. 8) at $z=0$ are slightly modified in order to set a uniform velocity profile at the lower section of the channel. Because the velocity is now imposed, the pressure is set free in the lower section, such that $\partial p_{g} / \partial \mathbf{n}=0$ (same condition implicitly set on solid walls). Note that imposing a velocity profile or the coupled pressure/velocity condition deduced from 
the Bernoulli relation (Eq. 8) in the entrance section of the channel for mixed convection was discussed in [55]. The depth of flow reversal is determined by the dimensionless length build on the hydraulic diameter of the channel and defined by $Z_{r}^{*}=\left(H-z_{r}\right) /(2 D)$ where $z_{r}$ designates the ordinate satisfying the condition $\partial w /\left.\partial x\right|_{x=D}=0$. In this section, the computations are performed with a $32 \times 128$ uniform grid, the same mesh as in reference [16]. Table 2 shows a good agreement with a maximal discrepancy of $5.3 \%$ for $R e=400$ and $R i=100$.

\section{Results}

\subsection{Comparisons with the experimental results by Webb and Hill [1]}

The experimental apparatus used by Webb and Hill [1] consisted in a vertical parallel plate channel of high $2 \mathrm{H}=30.4 \mathrm{~cm}$ formed by two walls, one heated and the other adiabatic (Fig. 1). The length of the heated section was $H=15.2 \mathrm{~cm}$ and unheated $H / 2=7.6 \mathrm{~cm}$ entry and exit lengths were added to minimize radiation losses with the cold ambient environment. The three wall spacings $(D=1.02 \mathrm{~cm}, 2.02 \mathrm{~cm}$ and $3.8 \mathrm{~cm})$, the three Ohmic dissipation fluxes at the heated wall $\left(q_{w}=235 \mathrm{~W} / \mathrm{m}^{2}, 300 \mathrm{~W} / \mathrm{m}^{2}\right.$ and $\left.375 \mathrm{~W} / \mathrm{m}^{2}\right)$ considered in the present subsection are those used for preparing Fig. 2 displayed in [1]. The data reported in these figures were digitalized and are illustrated in the first two figures discussed below. The first remark about the design of this experimental set-up, is that the upper extension has a significant effect on the flow, as it was demonstrated in others experimental or numerical works, $[40,42]$ for example.

The profiles of the temperature differences from the inlet temperature $T_{0}$ along the heated section are plotted in Fig. 2(a) for the three heating rates, a wall spacing of $D=2.02 \mathrm{~cm}$ and an emissivity of both vertical wall of $\epsilon=0.1$, as it was assumed by Webb and Hill [1]. These fluxes are not corrected for the conduction losses, estimated less than 1.8 percent of the Ohmic heating [1]. Since the ambient air temperature was not specified in [1], an inlet temperature of $T_{0}=290 \mathrm{~K}$ was retained. Note that lower $T_{0}$-values increase slightly the radiative heat losses from the heating section. However, computations carried out with $T_{0}=273 \mathrm{~K}$ did not shown significant changes in the results reported in Fig. 2(a), owing to the small view factors between the heated section and the fictitious black surfaces, formed by the openings at the channel bottom and top provided that the channel aspect ratio is large enough. When the wall heat flux increases, the temperature differences along the walls increases, as expected. The maximum temperature is predicted at the top end of the heated wall, and it is followed by an abrupt temperature decrease along the adiabatic section. As can be seen, the temperature profile are similar to those reported in [1] while the present numerical results show higher temperatures, all the more since the wall heat flux is increased. These discrepancies are attributed to the conduction to the polystyrene insulation and to the conduction in the aluminum foil used for covering the adiabatic top section which acts as a fin. This point was mentioned in [1].

Figure 2(b) illustrates the ratio of radiative heat flux to the heat flux dissipated in the heated section for $q_{w}=300 \mathrm{~W} / \mathrm{m}^{2}$ and three plate spacings. The net radiative heat flux is positive (radiative heat losses) over the heated and adiabatic top sections of the left-hand 
side (LHS) wall while is it negative along both the bottom adiabatic section of the LHS-wall, and the facing unheated wall. The $q_{r}(z) / q_{w}$ profiles are closely similar to those reported in [1]. However, the discrepancies increase as the spacing increases. It should be noted that the values provided in [1] were calculated from the temperature measurements (Fig. 2(a)) and, are therefore obviously smaller than those computed in the present study.

\subsection{Pure natural convection}

In order to show the importance of the effects on radiation, computations were firstly carried out for pure natural convection for various spacings and wall heat fluxes. The height of the one-sided heated vertical channel is the same as in the previous subsection [1]. The thermophysical properties of air at $T_{0}=290 \mathrm{~K}$ are as follows:

$$
\begin{gathered}
\rho_{0}=1.2174 \mathrm{~kg} / \mathrm{m}^{3}, \mu_{0}=1.8058 \cdot 10^{-5} \mathrm{~kg} /(\mathrm{m} \cdot \mathrm{s}), \beta_{0}=1 / T_{0} K^{-1} \\
c_{p_{0}}=1006.1 \mathrm{~J} /(\mathrm{kg} \cdot \mathrm{K}), k_{0}=0.025505 \mathrm{~W} /(\mathrm{m} \cdot \mathrm{K})
\end{gathered}
$$

These properties were used for calculating the reference value of the channel Rayleigh number reported in subsequent Tables and Figures. Since the height of the heated section was set to $H=0.152 \mathrm{~m}$, it follows that $R a^{*}=2.825 \cdot 10^{10} q_{w} D^{5}$. Note that all computations were carried out for variable thermophysical properties based on the polynomial expressions given in [46].

At section $z=H / 2$ (inlet of the heated section), the vertical velocity turns from a parabolic profile (developing flow) into an almost flat profile as the wall spacing increases, while the flow rate increases first with $D$ and then decreases to, apparently, reaches an asymptotic value, as shown in Table 3. The vertical velocity and temperature profiles at the outlet of the heated section and at the outlet of the channel are plotted in Figs. 3 and 4 . As soon as the fluid enters the heated section, the velocity profile for spacings larger than $D=2.02 \mathrm{~cm}$ exhibits a boundary layer structure, well established at the outlet section of the heated wall, as shown in Fig. 3(a), the maximum vertical velocity being almost independent of the spacing. On the other hand, negative velocities are seen for $D \geq 2.02 \mathrm{~cm}$. That demonstrates the existence of flow reversals whose extends increase with the spacing. This pocket of downflow and recirculation was revealed experimentally for the first time by Sparrow et al. [17], with water as the working fluid. The temperature profiles reported in Fig. 3(b) are typical of a boundary layer flow along a single plate for $D \geq 1.4 \mathrm{~cm}$.

At the outlet channel section, the velocity profile (Fig. 3(c)) are not strongly modified, except for the increase in the downflow velocities. The main changes in the flow variables are seen from the temperature profiles (Fig. 3(d)) because the flow reversal induces an abrupt transition to the surroundings temperature.

The distributions of the temperature differences from the inlet temperature $T_{0}$ along the two vertical walls are plotted in Fig. 4 for a heating rate of $q_{w}=300 \mathrm{~W} / \mathrm{m}^{2}$ and wall spacings ranging from $D=1.02 \mathrm{~cm}$ to $D=7.1 \mathrm{~cm}$ (i.e. $945 \leq R a^{*} \leq 1.54 \cdot 10^{7}$ ). When the wall spacing augments, the temperatures of the heated wall increase and reach their maximum values for $D \approx 2.3 \mathrm{~cm}$, showing that the single-plate limit is approached. The bottom adiabatic section is almost uniformly at $T_{0}$ while the temperature of the top adiabatic 
section decreases abruptly from a maximum value almost independent of $D$ provided that $D \geq 1 \mathrm{~cm}: T_{\max } \approx 346 \mathrm{~K}$. This result indicates that the minimum heat transfer coefficient, $h_{\min }=q_{w} /\left(T_{\max }-T_{0}\right)$, does not depend on $D$. On the other hand, the average temperature of the heated section increases first with $D$ and reaches then an almost constant value for $D \geq 2.3 \mathrm{~cm}$. Therefore, the mean heat transfer coefficient, calculated by using either Eq. 15 or Eq. 18, decreases at first with the wall spacing. For $1.02 \mathrm{~cm} \leq D \leq 2.3 \mathrm{~cm}$, the adiabatic RHS-wall is heated by the flow of hot air at its upper part, and the decrease in its temperature as $D$ increases shows the transition to the asymptotic regime, for which the RHS-wall is uniformly at $T_{0}$.

Figure 5 shows the isotherms (left side) and streamlines (right side) for $D=2.02 \mathrm{~cm}$ ( $R a^{*}=$ $945)$ and $D=3.8 \mathrm{~cm}\left(R a^{*}=6.78 \cdot 10^{5}\right)$ in the case $q_{w}=300 \mathrm{~W} / \mathrm{m}^{2}$. The dimensional steamfunction $([\mathrm{kg} /(\mathrm{m} \cdot \mathrm{s})])$ for $2 \mathrm{D}$ and variable density flows is calculated from its definition as

$$
\rho u=\frac{\partial \psi}{\partial z}, \quad \rho w=-\frac{\partial \psi}{\partial x}
$$

In what follows, the plots of the streamlines are for a streamfunction value at the RHS wall arbitrarily set to zero. The dashed lines (negative $\psi$-values) are for the streamlines into the pocket of flow recirculation. The streamlines show boundary layer development along the heated wall and penetration of a downcoming flow along the RHS-wall. At the stagnation point located very close to the RHS-wall, the downward flow starts to merge into the flow coming from the inlet section. The formation of reversal flow results from air drawn in through the channel exit due to insufficient incoming air through the inlet. The size of the pocket-like streamlines increase with $R a^{*}$ (or $D$ ) and the penetration depth of the recirculating flow increases also. This result is in good agreement with the experimental and numerical studies by Sparrow et al. [17] for a one-sided heated vertical channel and by Kihm et al. [19] for isothermal vertical walls.

The recirculation pocket extends both downward and in width as $D$ increases, as illustrated in Fig. 5. The width $d_{\psi}$ of the recirculation at the outlet of the heated section $\left(d_{\psi}(3 H / 2)\right.$ at $z=3 H / 2$, Eq. 37) and at the channel outlet $\left(d_{\psi}(2 H)\right.$ at $z=2 H$, Eq. 36), and the penetration length $L_{p}$ are provided in Fig. 6 versus the wall spacing. These quantities are based on the location of the $\psi=0$ value of the streamfunction: the width of the circulation at any $z$-position is the distance between the $\psi=0$ streamline and the RHS-wall, and the penetration length is the distance from the outlet section at which the $\psi=0$ streamline separates from the RHS-wall (stagnation point). Figure 6(a) shows the increases in the recirculation width versus the wall spacing at the two aforementioned channel sections. It can be seen that the wall spacing for the onset of the recirculation appears to be very close to $D=1.5 \mathrm{~cm}$ and that the recirculation width increases almost linearly with $D$ (or as $R a^{* 1 / 5}$ ), and that $d_{\psi}(3 H / 2) \approx d_{\psi}(2 H)$ for $D \geq 4 \mathrm{~cm}$. The penetration length (Fig. $\left.6(\mathrm{~b})\right)$ increases sharply with $D$ just after the formation of a recirculating flow while a slow augmentation of $L_{p}$ is observed for $D \geq 4 \mathrm{~cm}$. This behavior is in fairly good agreement with that reported in Kihm et al. [19] for symmetrically isothermal walls and much smaller modified-Rayleigh numbers. 
The mass flow rate $\left(\dot{m}_{i n}\right.$, Eq. 34) through the inlet section is reported in Table 3 as a function of the wall spacing. As can be seen, $\dot{m}_{i n}$ reaches a maximum for $D \approx 1.7 \mathrm{~cm}$, and decreases thereafter. An optimal plate spacing for natural convection between an array of symmetrically heated plates was first demonstrated at the same time by Bar Cohen and Rohsenow [18] and Bejan [56], and discussed in details in a recent paper by Sun et al. [44]. The present results show that such an optimum exists also for asymmetrically heated channels, but no attempt at correlating this spacing with $R a^{*}$ was done for the specific case considered, because it is clear that it depends also on the length of the adiabatic upper section (chimney effect). However, the main difference with symmetric heating is that the reduction in the mass flow rate at the inlet section occurs almost simultaneously with the onset of a flow reversal at the top part of the channel. From the present computations for $q_{w}=300 \mathrm{~W} / \mathrm{m}^{2}$, it can be concluded that the optimal spacing is close to $D=0.75 \mathrm{~cm}$ if it is based on the $D$-value at which the mean and maximum temperatures of the heated section reach asymptotic values $\left(\bar{T} \approx 333 \mathrm{~K}, T_{\max } \approx 347 \mathrm{~K}\right)$.

From the values of the mass flow rate through the outlet section reported in Table $3, \dot{m}_{\text {out }}$ (Eq. 35), it can be seen that $\dot{m}_{\text {in }}=\dot{m}_{\text {out }}$ up to $D=1.4 \mathrm{~cm}$. Since $\dot{m}_{\text {out }}$ is the sum of $\dot{m}_{\text {in }}$ plus the upward recirculating flow, the difference $\dot{m}_{\text {out }}-\dot{m}_{\text {in }}$ characterizes the onset and intensity of the recirculation. From Table 3, it can be seen that $\dot{m}_{\text {out }}$ is almost constant for $D \geq 2.02 \mathrm{~cm}$ while $\dot{m}_{i n}$ decreases (see also Fig. 11). The effect of the recirculation is to concentrate the streamlines corresponding to the induced air flow at the entrance region $(\psi>0)$ and to provide enough cold air, suck in through the outlet section, in order to feed the boundary layer. Hence the rise in the boundary layer thickness as $D$ increases is due to the downcoming flow through the outlet section.

The present numerical results can be well fitted by the least square method with correlation coefficients greater than $r^{2}=0.99$. The correlations turned out to be:

$$
\begin{aligned}
\overline{N u}_{c, 1}=0.859 R a^{*^{0.183}}, \overline{N u}_{c, 2}=0.763 R a^{*^{0.188}}, \\
\overline{N u}_{1 / 2}=0.721 R a^{*^{0.189}}, \theta_{\max }=1.86 R a^{*^{-0.201}}
\end{aligned}
$$

The agreement between the above correlations and those reported from the experimental results by Webb and Hill [1] for the same configuration could considered as rather good. Table 4 gives a more realistic view about the discrepancies between these correlations for the lowest and highest Rayleigh numbers corresponding to both works. Note that the range of $R a^{*}$ considered is within the so-called single-plate regime. The first result is that the discrepancies increase with $R a^{*}$ from $2.9 \%$ to $13 \%$ for $\overline{N u}_{c, 1}$. The second result clearly indicates that the definition of the average heat transfer coefficient following Eq. (15) or Eq. (18) has a significant influence (up to $8 \%$ for $R a^{*}=10^{3}$ ). This finding was not mentioned previously. The differences between the experimentally [1] and numerically determined midheight Nusselt numbers are within the range $6.1 \%$ to $11 \%$ for $10^{3} \leq R a^{*} \leq 1.5 \cdot 10^{7}$. About the maximum heated wall temperature, the discrepancies with the experimental results from Manca et al. [50] can be attributed to different chimney effects in the two studies since the 
upper extension was larger than the channel width in [50].

\subsection{Effects of surface radiation on the flow field and heat transfer}

From the above result discussion (subsection 4.1), it is clear that radiative exchanges amongst surfaces have a significant effects on the flow field and heat transfer when the facing plates are submitted to imposed heat fluxes. Figure 7 shows the effect of radiation on the temperature distributions along the two vertical walls according to their emissivity, assumed equal for both walls. The selected case is $D=3.8 \mathrm{~cm}$ and $q_{w}=300 \mathrm{~W} / \mathrm{m}^{2}$, i.e. the largest configuration experimentally studied in [1]. As can be seen, the temperature of the heated surface decreases when the emissivity is increased, the opposite being predicted for the facing adiabatic surface whose temperature increases. Therefore, radiation modifies the flow field, and hence the wall convective heat transfer. This point raises some doubts about the interpretation of experimental results and derivation of Nusselt number correlations when the effects of radiation are not properly evaluated. For example, if the radiative exchanges amongst the surfaces can be calculated from the surface temperature measurements, the convective heat flux profiles can be easily obtained (provided that the conductive heat losses are accurately estimated). Nevertheless, the temperature distribution along the heated surface, used in the definition of the local heat transfer coefficient, results from the coupling between radiation and convection. Therefore, the heat transfer coefficient is overestimated for a surface cooled by radiation and underestimated if heated by radiation.

The effect of radiation on the vertical velocity profiles at three channel cross sections are seen in Figs. 8(a)-8(c). The increase in the velocities (and in flow rate because the density variations from the channel inlet are very small) with emissivity at the inlet section of the heated wall is clearly seen in Fig. 8(a). The reason is that the adiabatic RHS-wall becomes thermally active as soon as its temperature raises significantly under the effect of surface radiation. When the fluid proceeds upwards the boundary-layer like velocity profile along the heated wall turns into a boundary layer profile of an asymmetrically heated channel, as seen in 8(b) and 8(c) for $\varepsilon=1$. Furthermore, the flow reversal disappears for a wall emissivity as small as $\varepsilon=0.1$. The profiles of the temperature differences reported in Figs. $8(\mathrm{~d})-8(\mathrm{f})$ give another view of the decrease in the heated wall temperature, and increases of the fluid and adiabatic wall temperatures.

The net radiative heat flux distribution along the LHS-heated wall and RHS-adiabatic wall are drawn in Figs. 9(a) and $9(\mathrm{~b})$ for $\varepsilon=0.1$ and $\varepsilon=1$, respectively $(D=2.02 \mathrm{~cm}$, $\left.q_{w}=300 \mathrm{~W} / \mathrm{m}^{2}\right)$. Positive $q_{r}(z)$-values are for radiative cooling and negative ones for radiative heating. For both cases, the RHS-wall is heated by radiation over most of its surface and the heating rate increases sharply with emissivity. The bottom adiabatic part of the LHS-wall is heated by the radiation reflected by the RHS-wall, and cooled by radiation exchanges with the cold inlet section, assumed to be a black surface at $T_{0}=290 \mathrm{~K}$. Since the view factor between the inlet section and the bottom of the walls is rather small, the adiabatic bottom of the heated wall is always heated by radiation. This result is just specific to the small $D$-value considered. More significant is the relative magnitude of the radiative 
heat losses, $\bar{q}_{r}$, to the heating flux, $q_{w}$, along the heated section. For $\varepsilon=0.1$ (as in the experiments by Webb and Hill [1]), the average radiative heat flux is $\bar{q}_{r}=23 \mathrm{~W} / \mathrm{m}^{2}(7 \%$ of $q_{w}$ ) and reaches $\bar{q}_{r}=118 \mathrm{~W} / \mathrm{m}^{2}$ (more than $40 \%$ of $q_{w}$ ) for $\varepsilon=1$. As expected, the peaks in $q_{r}(z)$ correspond to the maximum in wall temperature. The results are that, both the average and maximum temperatures of the heated section decrease under the effects of surface radiation. We will consider in what follows the effect of surface radiation on the convective Nusselt number when introducing a decoupling between convective and radiative modes, as it is usually done when maximum surface temperatures do not exceed about $10 \%$ of the inlet gas temperature (in $K$ ).

Comparisons between the isothermal and streamline patterns shown in Fig. 5 for $\varepsilon=0$ and those in Fig. 10 for $D=2.02 \mathrm{~cm}$ and $3.8 \mathrm{~cm}$ clearly exhibit the large changes in the flow topology under the effects of surface radiation for $\varepsilon=0.1$ and $\varepsilon=1$. These patterns demonstrate that radiation exchanges lead to a modification in the thermal conditions from a one-wall heating to an asymmetric heating, with the disappearance of the flow reversal. The insulated wall being heated by radiation, the downcoming flow from the outlet section cannot persist as soon as the temperature of that wall exceeds just a little the surrounding temperature. That happens at very low emissivity of the walls. When the radiative heating of the insulated wall is large enough, a thermal boundary layer develops along the wall, and starts at a lower $z$-elevation than at the heated wall. These observations may explain why flow reversals are difficult to observe experimentally if the working fluid is a gas. On the other hand, the recent experiments conducted by Ospir et al. [20] with water as the working fluid and for the same boundary conditions as in the present study provide very reliable pictures on the flow reversal.

The variation of the mass flow rate reported in Fig. 11 for $q_{w}=300 \mathrm{~W} / \mathrm{m}^{2}$ indicates continuous increases in $\dot{m}_{i n}$ with the wall spacing for $\varepsilon=0.1$ and $\varepsilon=1$ since any downcoming air flow is predicted for both wall emissivities. On the other hand, the flow reversal discussed previously for $\varepsilon=0$ leads to a maximum in the flow rate at the inlet section (see Table 3) while the outcoming flow rate at the outlet section $\dot{m}_{\text {out }}$ (Eq. 35) is almost constant for $D \geq D_{\text {opt }}$, as seen in Fig. 11. The influence of surface radiation on the mean and maximum temperature of the heated section is reported in Table 5 for the minimum and maximum plate spacings considered in the present study. The overall effect is a reduction in both $\bar{T}$ and $T_{\max }$ as $\varepsilon$ increases. Such a reduction is in part due to the radiative exchanges with the outlet section assumed as a black surface at $T_{0}$. That explains why the effect of radiation is more important as the modified Rayleigh is increased. Here again, this result is specific to the cases considered because the variations in $R a^{*}$ are only due to changes in $D$ while the channel height is kept constant. Hence, it cannot be concluded that radiation effects increase with the Rayleigh number, as it has been assumed in many papers. 


\subsubsection{Correlations}

Owing to the present definition of the conduction-to-radiation parameter, $N_{R}=0.748$ for an inlet air temperature $T_{0}=290 \mathrm{~K}$ and $q_{w}=300 \mathrm{~W} / \mathrm{m}^{2}$. The radiative Nusselt is therefore of the order of magnitude of the convective Nusselt number for black surfaces. Note that the emissivity is not accounted for in the definition of $N_{R}$ : the influence of surface radiation is thus much smaller for low surface emissivities than it seems, just by considering only the $N_{R}$ value.

The convective and radiative Nusselt numbers defined by Eq. 29 are reported in Fig. 12. In comparison with $\varepsilon=0, \overline{N u}_{c, 2}$ is slightly modified for $\varepsilon=0.1$ while its reduction is more significant for $\varepsilon=1$. In that case $\overline{N u}_{r, 2}$ is of the order of $\overline{N u}_{c, 2}$. The combined radiation convection effect produces a reduction in the maximum temperature of the heated wall, all the more large since the plate spacing and the emissivity increase. For $q_{w}=300 \mathrm{~W} / \mathrm{m}^{2}$ and $\varepsilon=1$, that reduction lies between $16.0 \mathrm{~K}$ for $D=1.02 \mathrm{~cm}$ and $24.2 \mathrm{~K}$ for $D=7.1 \mathrm{~cm}$. The present computations lead to the following correlations

$$
\begin{gathered}
\overline{N u}_{c, 2}=0.753 R a^{*^{0.189}}, \quad \overline{N u}_{r, 2}=0.0279 R a^{*^{0.248}} \text { for } \varepsilon=0.1 \\
\overline{N u}_{c, 2}=0.759 R a^{*^{0.181}}, \quad \overline{N u}_{r, 2}=0.253 R a^{*^{0.232}} \text { for } \varepsilon=1 \\
\theta_{\text {max }}=1.88 R a^{*^{-0.207}} \text { for } \varepsilon=0.1, \quad \theta_{\max }=1.60 R a^{*^{-0.226}} \text { for } \varepsilon=1
\end{gathered}
$$

\subsection{On the procedures used for the determination of the convective heat transfer coefficient from temperature measurements}

As discussed in the previous sections, surface radiation has a strong influence on the flow field and makes difficult the observation of air flow reversals. Its effect on the total heat transfer rate at the heated wall is also significant when mirror like wall surfaces are not used in experimental set up. Since emissivities as low as $\varepsilon=0.05$ are difficult to maintain due to oxidation produced by the air flow (except if the wall are covered with gold films), most experiments conducted for different heat fluxes prescribed to the walls are for non negligible combined effects of surface radiation and natural convection. The usual ways employed to determine the convective heat transfer coefficient are first the evaluation of the radiative heat flux from the temperature measurements along the walls. The convective heat flux is then obtained, just by subtraction, i.e. $q_{c v}(z)=q_{w}-q_{r}(z)$ (provided that the conductive heat losses have been properly considered in the evaluation of $q_{w}\left(\right.$ i.e. $\left.q_{w}=q_{\text {Ohmic }}-q_{\text {conduction }}\right)$ ).

Such a procedure has been followed, for example, by Webb and Hill [1] and more recently by Krishnan et al. [36] who used a combined experimental and numerical approach. Since we are modeling the full coupled heat transfer modes, it is easy to proceed exactly as it was done by experimentalists. 
As an example, the case $D=2.02 \mathrm{~cm}$ and $q_{w}=300 \mathrm{~W} / \mathrm{m}^{2}$ is considered (note that the effects of radiative exchanges with the cold inlet or outlet sections are rather weak in that case). The coupled solutions are displayed in Fig. 10(a) $(\varepsilon=0.1)$ and in Fig. 10(b) $(\varepsilon=1)$. The heat transfer results for the three approximate approaches are summarized in Table 6 . The corresponding streamlines and isotherms are displayed in Figs. 13 when the radiative heat fluxes or temperature distributions computed from the coupled problem for $\varepsilon=0.1$ and $\varepsilon=1$, respectively, are used to generate the thermal boundary conditions on the heated section: $\bar{q}_{w}^{\prime}, T_{w}(z)$ or $\bar{T}$.

- $\bar{q}_{r}$ is subtracted from $q_{w}$ (rows $2 \mathrm{a}$ and $3 \mathrm{a}$ in Table 6 ).

First, the average radiative flux at the heated section is calculated from the coupled computations: $\bar{q}_{r}=19 \mathrm{~W} / \mathrm{m}^{2}$ for $\varepsilon=0.1$ and $\bar{q}_{r}=108 \mathrm{~W} / \mathrm{m}^{2}$ for $\varepsilon=1$.

Computations were carried out by setting $\varepsilon=0$ and $\bar{q}_{w}^{\prime}=q_{w}-\bar{q}_{r}$. The plots of the isotherms and streamlines reported in Figs. 13(a) and 13(d) exhibit large differences both between the true flow fields (Figs. 10(a) and 10(b)) while it resembles more or less those which are in the absence of surface radiation (i.e. for $q_{w}=300 \mathrm{~W} / \mathrm{m}^{2}$, row 1 in Table 6). Hence it could be assumed that this procedure leads to wrong calculations of the heat transfer rate. However, the overall convective heat transfer is weakly altered by the presence of a flow reversal, as it was also noted by Kihm et al. [19]. Comparisons between the $\bar{h}_{c, 1}, \bar{h}_{c, 2}$ and $T_{\max }$ reported in rows 1,2 and $2 \mathrm{a}$ for $\varepsilon=0.1$ show that the differences are within $1 \%$. Therefore the procedure followed by Webb and Hill [1] may be considered accurate. Similar comparisons between rows 3 and 3a for $\varepsilon=1$ show slightly larger differences, but less than $3 \%$. On the other hand, comparisons between rows 1, 3 and 3a demonstrate that a radiative heat flux correction is needed for black surfaces, since the departures decrease from $6 \%$ (without correction) to $3 \%$ (with correction).

- Temperatures at the heated section used as boundary conditions for the computations [36] (rows 2b-c and 3b-c in Table 6).

The temperature at the heated section is either the temperature distribution (Figs. 13(b) and 13(e)) or the average temperature (Figs. 13(c) and 13(f)) extracted from the coupled problem. For the four cases considered, the average temperatures of the adiabatic wall sections determined from the coupled problem are applied as boundary conditions [36]: at the RHS-wall $(\bar{T}=292.7 \mathrm{~K}$ and $\bar{T}=302.5 \mathrm{~K}$ for $\varepsilon=0.1$ and $\varepsilon=1$, respectively), at the bottom extension of the LHS-wall $(\bar{T}=290.4 \mathrm{~K}$ and $\bar{T}=290.8 K$ for $\varepsilon=0.1$ and $\varepsilon=1$, respectively), and at the top extension of the LHS-wall $(\bar{T}=319.0 \mathrm{~K}$ and $\bar{T}=309.0 \mathrm{~K}$ for $\varepsilon=0.1$ and $\varepsilon=1$, respectively). Note that the small increase in the average temperature of the RHS-wall for $\varepsilon=0.1$ is enough to almost suppress the flow reversal, and that the top extension is colder for $\varepsilon=1$ than for $\varepsilon=0.1$.

The streamlines and isotherms displayed in Figs. 13(b)-(c) and Figs. 13(e)-(f) show a better agreement with those reported in 10(a) and 10(b) than those obtained by 
subtracting the average radiative flux. However, the average temperature applied at the adiabatic wall may lead to the development of a thermal boundary layer from its bottom (see the isotherms in Figs. 13(e)-(f)).

The results reported in rows $2 \mathrm{~b}$ and $2 \mathrm{c}$ of Table 6 show that the mean heat transfer rate and maximum temperature do not differ much from the previous cases if the temperature distribution $\left(T_{w}(z)\right)$ applied at the heated section is that deduced from the coupled formulation. On the other hand, the use of the average temperature leads to more significant differences, both in the convective coefficient and in the maximum temperature. This result conflicts with the analysis reported in [36].

\subsection{Non-Boussinesq effects}

The influence of property variations on natural convection from a vertical isothermal, heated surface was experimentally investigated by Clausing and Kempka [57]. By using a cryogenic environment, the ratio of the absolute temperature of the wall to the ambient temperature of gaseous nitrogen was varied in the range $1<T_{w} / T_{0} \leq 2.6$. The experimental apparatus allowed to study parts of both laminar and turbulent regimes $\left(10^{7} \leq R a_{H} \leq 2 \cdot 10^{10}\right)$. It was shown that variable properties have virtually no influence in the laminar regime while cause large increases in heat transfer rates in the turbulent regime. Guo and Zhao [45] used laser speckle photographic techniques for studying laminar natural convection between two isothermal vertical parallel plates in air. The surface-to-ambient temperature difference varied from $15 \mathrm{~K}$ to $530 \mathrm{~K}$, and the spacing of the $10 \mathrm{~cm}$-high plates was between $3 \mathrm{~mm}$ and $20 \mathrm{~mm}$. The experiments showed that the effects of variable properties are much larger than on a single plate. The subsequent numerical study by Guo and Wu [58] based on a parabolic formulation indicated strongest effects for small spacings and large values of the non-Boussinesq parameter. From these two studies, it was also shown that, unlike for the constant property case, the mass flow rate exhibits a nonmonotonic change with a maximum whose amplitude depends both on the spacing and heating rate. This question was numerically revisited by Zamora and Hernández [59] who used an elliptic formulation for an asymmetric heated channel with prescribed temperature at the hot wall and unheated facing wall. They showed in particular that variable property effects produce an important reduction of the recirculation region. It should be noted that experiments and computations showing large variable property effects on the flow patterns were carried out for very high temperature differences between the inlet and wall temperatures (up to $7 \times T_{0}$ ). Hence, conclusions about large variable property effects must be cautiously taken into consideration for practical applications dealing with cooling of electronic equipments or non-concentrated solar collectors, for example.

In what follows, results obtained from the variable property formulation are compared with those derived by invoking the Boussinesq approximation. One of the reasons is that Webb and Hill [1] suggested that the use of local properties is more appropriate for correlating local heat transfer data despite the maximum measured temperature differences between the 
bottom and top ends of the heated section were much smaller than in the work by Clausing and Kempka [57].

For the range of $R a^{*}$ investigated, the influence of $D$ (or $A$ ) was found negligible. The reason is that the maximum wall temperature is almost insensitive to $D$ provided that $D \geq 1 \mathrm{~cm}$, as shown in subsection 4.2. Therefore, we discuss only results obtained for $D=2.02 \mathrm{~cm}$. In order to focus on the influence of variable property effects, pure natural convection is considered. The wall heat flux was varied from $q_{w}=375 \mathrm{~W} / \mathrm{m}^{2}$ to $q_{w}=$ $1400 \mathrm{~W} / \mathrm{m}^{2}\left(3.56 \cdot 10^{4} \leq R a^{*} \leq 1.33 \cdot 10^{5}\right)$. The non-Boussinesq parameter was thus in the range $1.02 \leq \gamma \leq 3.82$, and the corresponding maximal wall temperature was found in the range $358 \mathrm{~K} \leq T_{\max } \leq 489 \mathrm{~K}$, i.e. $\Delta T_{\max } / T_{0}$ between 0.23 and 0.69 , larger than the usual 0.1-limit for the validity of the Boussinesq approximation. The dynamical viscosity and thermal conductivity of mixtures of ideal polyatomic gases increase with temperature, roughly as the absolute temperature to the power 0.6 to 1.0, while the density decreases as $T^{-1}$ (the pressure effects being negligibly small, see Eq. 4). Therefore, the opposite effects on viscous drag and buoyancy may produce non-monotonic and unpredictable variations of the flow rate with increasing the maximum temperature difference.

Figure 14 shows that the non-Boussinesq effects have a weak influence on the flow patterns near the heated wall for $q_{w}=375 \mathrm{~W} / \mathrm{m}^{2}$, and that the flow rate at the inlet section is slightly increased (about 9\%) for $q_{w}=1400 \mathrm{~W} / \mathrm{m}^{2}$ due to variable property effects. The most salient result is about the reduction of the size of the recirculation region (width and penetration length). This reduction and the increase in $\dot{m}_{i n}$ are linked, as discused previously (Figs. 6 and 11). Concerning the heat transfer results, it has been found that the influence of variables properties is almost null for the smallest heat flux considered $\left(q_{w}=235 \mathrm{~W} / \mathrm{m}^{2}\right)$ while the changes in average and maximum temperatures of the heated section were about $1 \%$ for the highest $q_{w}$-value $\left(q_{w}=1400 \mathrm{~W} / \mathrm{m}^{2}\right)$. Hence it can be concluded that the Nusselt number correlations reported previously would have been identical if the Boussinesq approximation has been invoked. As a result, taking variable properties effects into consideration for natural convection of air is not required for maximum increase in temperature less than about $200 \mathrm{~K}$. These trends are in general agreement with the results discussed by Zamora et Hernández [59] for asymmetric isothermal channels.

\section{Conclusion}

The combined effects of surface radiation and air natural convection in vertical, asymmetrically heated plate channels have been numerically investigated thanks to an improved mathematical formulation in which the temperature variations of all thermophysical properties are accounted for. The present formulation does not imply excessive increases in the computational costs. Extensive comparisons with the experimental results by Webb et Hill [1] have been carried out and heat transfer correlations for pure natural convection as well as for combined effects are provided. The agreement with the experimental results is fairly good and the onset of flow reversal for pure natural convection has been revisited. 
The study leads to the following conclusions:

- The onset of flow reversal is delayed upon the effect of surface radiation. Mirror like wall surfaces are required to observe experimentally flow recirculations when using air as the working fluid. The effect of the plate spacing on the formation of a recirculating flow and on the mass flow rates through both inlet and outlet sections has been analyzed in detail. The large feeding of the boundary layer along the heated wall due the penetration of a downcoming flow along the opposite wall has been quantitatively explored.

- The radiation contribution is to increase the temperatures of the adiabatic wall facing the heated section, leading to prevent the occurrence of down flow for wall emissivity as small as $\varepsilon=0.1$. Radiation enhances the cooling of the heated wall and its contribution to the total heat transfer rate is significant. For black surface at room temperatures, the heat transfer by radiation is of the order of that by natural convection.

- Procedures generally used which consist in subtracting the radiative contribution to the heat flux applied to the hot surface, $q_{w}$, have been examined. If the radiative heat flux distribution or the average radiative heat flux is subtracted from $q_{w}$, the coupled (i.e. $\varepsilon \neq 0$ ) and uncoupled (i.e. $\varepsilon=0$ ) numerical solutions lead to quite accurate predictions of the average convective heat transfer coefficient and maximum wall temperature. However, a flow reversal is predicted in the uncoupled solutions while it is not for the coupled solutions. If the measured temperature distribution along the heated section is used as the thermal boundary condition in a combined experimental and numerical approach, the heat transfer predictions are also quite accurate. On the other hand, large discrepancies are found when the average temperature of the heated section is employed as thermal boundary condition.

- Since the maximum temperature of the heated wall is a priori unknown for prescribed wall heat flux, a weakly compressible formulation for ideal gas has been used in carrying out the computations. This point was raised in [1] since maximum wall temperatures $50 \mathrm{~K}$ above the inlet temperature were measured. It could be much greater for higher wall heat fluxes or smaller plate spacings. The comparisons between the usual Boussinesq formulation and fully variable fluid properties (including density in the transport terms) showed that negligible influence of the variable property effects for surface-to-ambient temperature difference up to $200 K$.

- In order to allow the check of the numerical results reported in the Tables discussed herein, all the required data are fully provided. 


\section{REFERENCES}

[1] B. Webb, D. Hill, High Rayleigh number laminar natural convection in an asymmetrically heated vertical channel, Journal of Heat Transfer 111 (1989) 649-656.

[2] W. Elenbaas, Heat dissipation of parallel plates by free convection, Physica 9 (1) (1942) 1-28.

[3] J. Bodoia, J. Osterle, The development of free convection between heated vertical plates, Journal of Heat Transfer 84 (1962) 40-44.

[4] W. Aung, Fully developed laminar free convection between vertical plates heated asymmetrically, International Journal of Heat and Mass Transfer 15 (8) (1972) 1577-1580.

[5] W. Aung, L. Fletcher, V. Sernas, Developing laminar free convection between vertical flat plates with asymmetric heating, International Journal of Heat and Mass Transfer 15 (11) (1972) 2293-2304.

[6] R. Wirtz, R. Stutzman, Experiments on free convection between vertical plates with symmetric heating, Journal of Heat Transfer 104 (1982) 501-507.

[7] C. Olsson, Prediction of Nusselt number and flow rate of buoyancy driven flow between vertical parallel plates, Journal of Heat Transfer 126 (2004) 97-104.

[8] Q. Lu, S. Qiu, G. Su, W. Tian, Z. Ye, Experimental research on heat transfer of natural convection in vertical rectangular channels with large aspect ratio, Experimental Thermal and Fluid Science 34 (1) (2010) 73-80.

[9] B. Buonomo, O. Manca, Natural convection slip flow in a vertical microchannel heated at uniform heat flux, International Journal of Thermal Sciences 49 (8) (2010) 1333-1344.

[10] B. Buonomo, O. Manca, Transient natural convection in a vertical microchannel heated at uniform heat flux, International Journal of Thermal Sciences 56 (2012) 35-47.

[11] W. Aung, G. Worku, Theory of fully developed, combined convection including flow reversal, Journal of Heat Transfer 108 (2) (1986) 485-488.

[12] W. Aung, G. Worku, Developing flow and flow reversal in a vertical channel with asymmetric wall temperatures, Journal of Heat Transfer 108 (2) (1986) 229-304.

[13] C. Cheng, H. Kou, W. Huang, Flow reversal and heat transfer of fully developed mixed convection in vertical channels, Journal of Thermophysics and Heat Transfer 4 (3) (1990) 375-383.

[14] C. Cheng, S. Huang, W. Aung, Enhancement of flare method for predicting buoyancy-induced flow reversal in vertical ducts via parabolic model, Numerical Heat Transfer 31 (3) (1997) 327-345.

[15] Y. Jeng, J. Chen, W. Aung, On the reynolds-number independence of mixed convection in a vertical channel subjected to asymmetric wall temperatures with and without flow reversal, International Journal of Heat and Fluid Flow 13 (4) (1992) 329-339.

[16] C. Yang, D. Jeng, K. Yih, C. Gau, W. Aung, Numerical and analytical study of reversed flow and heat transfer in a heated vertical duct, Journal of Heat Transfer 131 (7) (2009) 072501 (9 pages).

[17] E. Sparrow, G. Chrysler, L. Azevedo, Observed flow reversals and measured-predicted nusselt numbers for natural convection in a one-sided heated vertical channel, Journal of Heat Transfer 106 (1984) $325-332$.

[18] A. Bar-Cohen, W. Rohsenow, Thermally optimum spacing of vertical, natural convection cooled, parallel plates, Journal of Heat Transfer 106 (1) (1984) 116-123.

[19] K. Kihm, J. Kim, L. Fletcher, Onset of flow reversal and penetration length of natural convective flow between isothermal vertical walls, Transactions American Society of Mechanical Engineers Journal of Heat Transfer 117 (1995) 776-776.

[20] D. Ospir, C. Popa, C. Chereches, G. Polidori, S. Fohanno, Flow visualization of natural convection in a vertical channel with asymmetric heating, International Communications in Heat and Mass Transfer 39 (4) (2012) 486-493.

[21] Y. Yamada, Combined radiation and free convection heat transfer in a vertical channel with arbitrary wall emissivities, International Journal of Heat and Mass Transfer 31 (2) (1988) 429-440.

[22] G. Colomer, M. Costa, R. Consul, A. Oliva, Three-dimensional numerical simulation of convection and radiation in a differentially heated cavity using the discrete ordinates method, International Journal of Heat and Mass Transfer 47 (2) (2004) 257-269.

[23] B. Mondal, X. Li, Effect of volumetric radiation on natural convection in a square cavity using lattice 
Boltzmann method with non-uniform lattices, International Journal of Heat and Mass Transfer 53 (2122) (2010) 4935-4948.

[24] C. Balaji, S. Venkateshan, Interaction of surface radiation with free convection in a square cavity, International Journal of Heat and Fluid Flow 14 (3) (1993) 260-267.

[25] C. Balaji, S. Venkateshan, Correlations for free convection and surface radiation in a square cavity, International Journal of Heat and Fluid Flow 15 (3) (1994) 249-251.

[26] N. Ramesh, S. Venkateshan, Effect of surface radiation on natural convection in a square enclosure, Journal of Thermophysics and Heat Transfer 13 (3) (1999) 299-301.

[27] E. Ridouane, M. Hasnaoui, A. Campo, Effects of surface radiation on natural convection in a rayleighbenard square enclosure: steady and unsteady conditions, Heat and Mass Transfer 42 (3) (2006) 214225 .

[28] M. Gad, C. Balaji, Effect of surface radiation on rbc in cavities heated from below, International Communications in Heat and Mass Transfer 37 (10) (2010) 1459-1464.

[29] O. Manca, S. Nardini, Experimental investigation of radiation effects on natural convection in horizontal channels heated from above, Journal of Heat Transfer 131 (6) (2009) 062503 (10 pages).

[30] H. Bouali, A. Mezrhab, H. Amaoui, M. Bouzidi, Radiationnatural convection heat transfer in an inclined rectangular enclosure, International Journal of Thermal Sciences 45 (6) (2006) 553-566.

[31] G. Lauriat, G. Desrayaud, Effect of surface radiation on conjugate natural convection in partially open enclosures, International Journal of Thermal Sciences 45 (4) (2006) 335-346.

[32] A. Mezrhab, H. Bouali, H. Amaoui, M. Bouzidi, Computation of combined natural-convection and radiation heat-transfer in a cavity having a square body at its center, Applied Energy 83 (9) (2006) 1004-1023.

[33] H. Sun, E. Chénier, G. Lauriat, Effect of surface radiation on the breakdown of steady natural convection flows in a square, air-filled cavity containing a centered inner body, Applied Thermal Engineering 31 (6-7) (2011) 1252-1262.

[34] J. Carpenter, D. Briggs, V. Sernas, Combined radiation and developing laminar free convection between vertical flat plates with asymmetric heating, Journal of Heat Transfer 98 (1976) 95-100.

[35] O. Manca, V. Naso, Experimental analysis of natural convection and thermal radiation in vertical channels, ASME HTD 145 (1990) 13-21.

[36] A. Krishnan, B. Premachandran, C. Balaji, S. Venkateshan, Combined experimental and numerical approaches to multi-mode heat transfer between vertical parallel plates, Experimental Thermal and Fluid Science 29 (1) (2004) 75-86.

[37] D. Naylor, J. Floryan, J. Tarasuk, A numerical study of developing free convection between isothermal vertical plates, Journal of Heat Transfer (Transactions of the ASME, Series C) 113 (3) (1991) 620-626.

[38] B. Morrone, A. Campo, O. Manca, Optimum plate separation in vertical parallelplate channels for natural convective flows: incorporation of large spaces at the channel extremes, International Journal of Heat and Mass Transfer 40 (5) (1997) 993-1000.

[39] N. Anand, S. Kim, L. Fletcher, The effect of plate spacing on free convection between heated parallel plates, Journal of Heat Transfer (Transactions of the ASME, Series C) 114 (2) (1992) 515-518.

[40] A. Auletta, O. Manca, Heat and fluid flow resulting from the chimney effect in a symmetrically heated vertical channel with adiabatic extensions, International Journal of Thermal Sciences 41 (12) (2002) 1101-1111.

[41] A. Andreozzi, B. Buonomo, O. Manca, Numerical study of natural convection in vertical channels with adiabatic extensions downstream, Numerical Heat Transfer, Part A 47 (8) (2005) 741-762.

[42] A. Andreozzi, B. Buonomo, O. Manca, Thermal and fluid dynamic behaviors in symmetrical heated channel-chimney systems, International Journal of Numerical Methods for Heat \& Fluid Flow 20 (7) (2010) 811-833.

[43] T. Bello-Ochende, A. Bejan, Optimal spacings for mixed convection, Journal of Heat Transfer 126 (2004) 956-962.

[44] H. Sun, R. Li, E. Chénier, G. Lauriat, J. Padet, Optimal plate spacing for mixed convection from an array of vertical isothermal plates, Int. J. Therm. Sci. 55 (2012) 16-30. 
[45] Z. Guo, S. Y.Z., X. Zhao, Experimental investigation on natural convection in channel by laser speckle photography, Experimental Thermal and Fluid Science 4 (5) (1991) 594-600.

[46] D. Lide, H. Kehiaian, CRC handbook of thermophysical and thermochemical data, Vol. 1, CRC, 1994.

[47] H. Sun, G. Lauriat, X. Nicolas, Natural convection and wall condensation or evaporation in humid air-filled cavities subjected to wall temperature variations, International Journal of Thermal Sciences 50 (5) (2011) 663-679.

[48] M. Modest, Radiative heat transfer, 2nd Edition, Academic Press, 2003.

[49] N. Bianco, L. Langellotto, O. Manca, S. Nardini, Radiative effects on natural convection in vertical convergent channels, International Journal of Heat and Mass Transfer 53 (17) (2010) 3513-3524.

[50] O. Manca, M. Musto, V. Naso, Experimental analysis of asymmetrical isoflux channel-chimney systems, International Journal of Thermal Sciences 42 (9) (2003) 837-846.

[51] E. Chénier, R. Eymard, O. Touazi, Numerical results using a colocated finite volume scheme on unstructured grids for incompressible fluid flows, Numerical Heat Transfer Part B 49 (3) (2006) 259-276.

[52] O. Touazi, E. Chénier, R. Eymard, Simulation of natural convection with the collocated clustered finite volume scheme, Computers \& Fluids 37 (2008) 1138-1147.

[53] R. Li, Numerical simulations of natural or mixed convection in vertical channels - Comparisons of level-set numerical schemes for the modeling of immiscible incompressible fluid flows, Ph.D. thesis, Université Paris-Est Marne-la-Vallée, France (2012).

[54] K. Goda, A multistep technique with implicit difference schemes for calculating two- or threedimensional cavity flows, Journal of Computational Physics 30 (1979) 76-95.

[55] H. Sun, R. Li, E. Chénier, G. Lauriat, On the modeling of aiding convection in vertical channels, Heat Mass Transfer 48 (7) (2012) 1125-1134.

[56] A. Bejan, Convection heat transfer, John Wiley and Sons Inc., New York, NY, 1984.

[57] A. Clausing, S. Kempka, The influences of property variations on natural convection from vertical surfaces, Journal of Heat Transfer 103 (1981) 609-612.

[58] Z. Guo, X. Wu, Thermal drag and critical heat flux for natural convection of air in vertical parallel plates, Journal of Heat Transfer 115 (1) (1993) 124-129.

[59] B. Zamora, J. Hernández, Influence of variable property effects on natural convection flows in asymmetrically-heated vertical channels, International Communications in Heat and Mass Transfer 24 (8) (1997) 1153-1162. 


\section{List of Tables}

1 Natural convection. Effect of the grid size for (a) $D=7.1 \mathrm{~cm}$, (b) $D=1.71 \mathrm{~cm}$ $\left(q_{w}=300 \mathrm{~W} / \mathrm{m}^{2}\right) \ldots \ldots \ldots \ldots \ldots \ldots \ldots \ldots$

2 Dimensionless length of the reversal flow $Z_{r}^{*}$ as a function of the Reynolds number and Richardson number $R i=R a /\left(\operatorname{Pr} R e^{2}\right)$. . . . . . . . . . .

3 Natural convection. Mass flow rate as a function of the wall-spacing $\left(q_{w}=\right.$ $\left.300 \mathrm{~W} / \mathrm{m}^{2}\right) \ldots \ldots \ldots \ldots \ldots \ldots$

4 Natural convection. Comparisons between the present results with previous correlations $[1,50]$ for the range of channel Rayleigh numbers considered (single-plate regime). . . . . . . . . . . . . . . . .

5 Comparisons between the average and maximum heated wall temperatures $\left(q_{w}=300 \mathrm{~W} / \mathrm{m}^{2}\right) \ldots \ldots \ldots \ldots \ldots \ldots$

6 Comparisons between the different procedures used for the convective part $(D=2.02 \mathrm{~cm})$. N. C.: pure natural convection, Coupled: combined radiation and natural convection, ${ }^{*}$ denotes an output from the temperature distribution, $R a^{*}$ are based on the average wall heat flux given in column $4 . . .$.

\section{List of Figures}

1 Geometry of the parallel-plate channel.

2 Variations of (a) the heated wall temperature and (b) the local radiative flux to the imposed wall heat flux versus $z / H(\epsilon=0.1)$. Symbols are experimental data from Webb and Hill [1].

(a) Variations of the heated wall temperature for the three heating rates $(D=2.02 \mathrm{~cm}) \ldots \ldots \ldots \ldots \ldots \ldots$

(b) Variations of the local radiative flux for three wall spacings ( $q_{w}=$

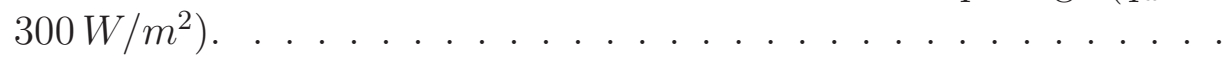

3 Natural convection. (a), (c) Vertical velocity profiles and (b), (d) temperature according to the wall spacing $\left(q_{w}=300 \mathrm{~W} / \mathrm{m}^{2}\right) \ldots \ldots \ldots \ldots$

(a) Vertical velocity, $z=3 H / 2 \ldots \ldots \ldots \ldots$

(b) Temperature, $z=3 H / 2 \ldots \ldots \ldots \ldots$

(c) Vertical velocity, $z=2 H \ldots \ldots \ldots \ldots$

(d) Temperature, $z=2 H \ldots \ldots \ldots \ldots \ldots$

4 Natural convection. Temperature distribution along the vertical channel walls according to the wall spacing $\left(q_{w}=300 \mathrm{~W} / \mathrm{m}^{2}\right)$. The open circle symbols are for the adiabatic wall. . . . . . . . . . . . . . . . .

$5 \quad$ Natural convection. (a) Streamlines $\psi_{i} /[\mathrm{g} /(\mathrm{m} \cdot \mathrm{s})]=0.25 \times i$ and (b) isotherms $T_{i} /[K]=290+5 \times i$ for $D=2.02 \mathrm{~cm}$ and $D=3.8 \mathrm{~cm}\left(q_{w}=300 \mathrm{~W} / \mathrm{m}^{2}\right)$. The horizontal scales are enlarged by $50 \%$. . . . . . . . . . . . . . 40

(a) Streamlines ....................... 40

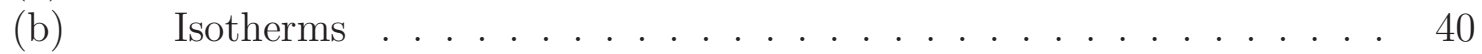


6 Natural convection. (a) Recirculation widths and (b) penetration length $\left(L_{p}\right)$ versus the channel width $\left(q_{w}=300 \mathrm{~W} / \mathrm{m}^{2}\right) \ldots \ldots \ldots$. . . . . . . . 40

(a) Recirculation widths . . . . . . . . . . . . . . . 40

(b) Penetration length ...................... 40

7 Variation of the heated wall temperature with height according to the emissivity of the vertical walls $\left(D=3.8 \mathrm{~cm}\right.$ and $\left.q_{w}=300 \mathrm{~W} / \mathrm{m}^{2}\right)$. The open circle symbols are for the adiabatic wall. . . . . . . . . . . . . .

8 Vertical velocity profiles (a) at the inlet of the heated wall section $(z=H / 2)$, (b) at the outlet of the heated wall section $(z=3 H / 2)$, (c) at the outlet section of the channel $(z=2 H)$ and temperature gap profiles (d) at the inlet of the heated wall section $(z=H / 2)$, (e) at the outlet of the heated wall section $(z=3 H / 2)$, (f) at the outlet section of the channel $(z=2 H)$ as a function of the emissivity of the vertical walls $\left(D=3.8 \mathrm{~cm}, q_{w}=300 \mathrm{~W} / \mathrm{m}^{2}\right)$. (a) Vertical velocity profile, $z=H / 2 \ldots \ldots$. . . . . . 42

(b) Vertical velocity profile, $z=3 H / 2 \ldots \ldots \ldots$. . . . . . . 42

(c) Vertical velocity profile, $z=2 H \ldots \ldots$. . . . . . . . 42

(d) Temperature increase, $z=H / 2 \ldots \ldots \ldots \ldots$. . . . . . . 42

(e) Temperature increase, $z=3 H / 2 \ldots \ldots \ldots \ldots$. . . . . . . 42

(f) Temperature increase, $z=H \ldots \ldots \ldots . \ldots . \ldots . \ldots . \ldots 42$

9 Distribution of the net radiative heat flux densities along (1) the heated wall and (2) along the adiabatic wall, with (a) $\epsilon=0.1$ and (b) $\epsilon=1(D=2.02 \mathrm{~cm}$ and $\left.q_{w}=300 \mathrm{~W} / \mathrm{m}^{2}\right) \ldots \ldots \ldots \ldots$. . . . . . . . . . . . . . . . . . . . . .

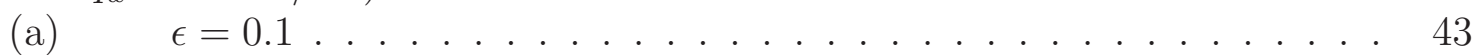

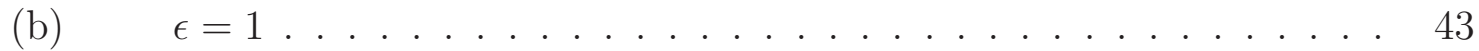

10 Effect of surface radiation on streamlines $\psi_{i} /[\mathrm{g} /(\mathrm{m} \cdot \mathrm{s})]=0.25 \times i$ (left) and isotherms $T_{i} /[K]=290+5 \times i$ (right) for (a) $D=2.02 \mathrm{~cm}, \epsilon=0.1$, (b) $D=2.02 \mathrm{~cm}, \epsilon=1$, (c) $D=3.8 \mathrm{~cm}, \epsilon=0.1$, (d) $D=3.8 \mathrm{~cm}, \epsilon=1$ $\left(q_{w}=300 \mathrm{~W} / \mathrm{m}^{2}\right)$. The horizontal scales are enlarged by $50 \%$. . . . . . . 43

(a) $\quad D=2.02 \mathrm{~cm}, \epsilon=0.1 \ldots \ldots \ldots \ldots \ldots \ldots$

(b) $\quad D=2.02 \mathrm{~cm}, \epsilon=1 \ldots \ldots \ldots \ldots \ldots$

(c) $\quad D=3.8 \mathrm{~cm}, \epsilon=0.1 \ldots \ldots \ldots \ldots \ldots$

(d) $\quad D=3.8 \mathrm{~cm}, \epsilon=1 \ldots \ldots \ldots \ldots \ldots$

11 Mass flow rate $\dot{m}_{\text {in }}$ versus the wall spacing for three wall emissivities and $\dot{m}_{\text {out }}$ for $\epsilon=0$. Mass flow rates $\dot{m}_{\text {out }}=\dot{m}_{\text {in }}$ for $\epsilon=0.1$ and $\epsilon=1\left(q_{w}=300 \mathrm{~W} / \mathrm{m}^{2}\right)$.

12 Radiative $\left(\overline{N u}_{r, 2}\right)$ and convective $\left(\overline{N u}_{c, 2}\right)$ Nusselt numbers (eq. 30) versus the channel Rayleigh number for two emissivities of the vertical walls $\left(q_{w}=\right.$

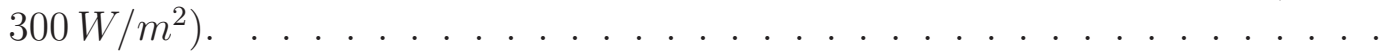

13 Natural convection. Streamlines $\psi_{i} /[\mathrm{g} /(\mathrm{m} \cdot \mathrm{s})]=0.5 \times i$ (left) and isotherms $T_{i} /[K]=290+5 \times i$ (right) with (a), (d) imposed wall fluxes $\bar{q}_{w}^{\prime}$ and adiabatic surfaces, (b), (e) $T_{w}(z)$ and average temperatures on other walls and (c), (f) imposed average temperatures $\bar{T}_{w}$ and average temperatures on other walls $(D=2.02 \mathrm{~cm})$. The horizontal scales are enlarged by $50 \%$. . . . . . . . 45

(a) $\quad \bar{q}_{w}^{\prime}=281 \mathrm{~W} / \mathrm{m}^{2}$ and adiabatic surfaces (Tab. 6, row 2a) . . . . 45 
(b) $\quad T_{w}(z)$ and average temperatures on other walls (Tab. 6, row 2b).

(c) $\quad \bar{T}_{w}=332.8 \mathrm{~K}$ and average temperatures on other walls (Tab. 6, row

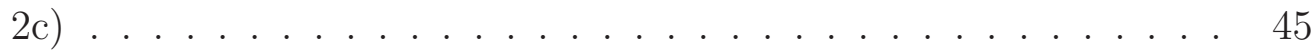

(d) $\quad \bar{q}_{w}^{\prime}=192 \mathrm{~W} / \mathrm{m}^{2}$ and adiabatic surfaces (Tab. 6, row 3a) . . . . 45

(e) $\quad T_{w}(z)$ and average temperatures on other walls (Tab. 6, row 3b) . 45

(f) $\quad \bar{T}_{w}=321.0 \mathrm{~K}$ and average temperatures on other walls (Tab. 6, row

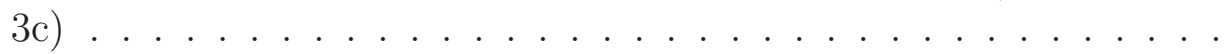

14 Natural convection. Streamlines $\psi_{i} /[\mathrm{g} /(\mathrm{m} \cdot \mathrm{s})]=0.5 \times i$ (left) and isotherms $T_{i} /[K]=290+20 \times i$ (right) with (a) $q_{w}=375 \mathrm{~W} / \mathrm{m}^{2}$ and the Boussinesq approximation, (b) $q_{w}=375 \mathrm{~W} / \mathrm{m}^{2}$ and the low Mach number approximation, (c) $q_{w}=1400 \mathrm{~W} / \mathrm{m}^{2}$ and the Boussinesq approximation, (d) $q_{w}=1400 \mathrm{~W} / \mathrm{m}^{2}$ and the low Mach number approximation $(D=2.02 \mathrm{~cm})$. The horizontal scales are enlarged by $50 \% \ldots \ldots \ldots \ldots$. . . . . . . . . . . . . . . . 46

(a) $\quad q_{w}=375 \mathrm{~W} / \mathrm{m}^{2}$, Boussinesq approximation . . . . . . . . . 46

(b) $\quad q_{w}=375 \mathrm{~W} / \mathrm{m}^{2}$, low Mach number approximation . . . . . . . . 46

(c) $\quad q_{w}=1400 \mathrm{~W} / \mathrm{m}^{2}$, Boussinesq approximation . . . . . . . . . 46

(d) $\quad q_{w}=1400 \mathrm{~W} / \mathrm{m}^{2}$, low Mach number approximation . . . . . . 46 


\section{TABLES}

(a) $D=7.1 \mathrm{~cm}, q_{w}=300 \mathrm{~W} / \mathrm{m}^{2}, R a^{*}=1.52 \cdot 10^{7}$

\begin{tabular}{c|c|c|c|c|c|c}
$n_{x} \times n_{z}$ & $\begin{array}{c}\dot{m}_{\text {in }} \\
{[\mathrm{g} /(\mathrm{m} \cdot \mathrm{s})]}\end{array}$ & $\begin{array}{c}\dot{m}_{\text {out }} \\
{[\mathrm{g} /(\mathrm{m} \cdot \mathrm{s})]}\end{array}$ & $\begin{array}{c}d_{\psi}(2 \mathrm{H}) \\
{[\mathrm{cm}]}\end{array}$ & $\begin{array}{c}d_{\psi}(3 \mathrm{H} / 2) \\
{[\mathrm{cm}]}\end{array}$ & $\begin{array}{c}\bar{h}_{c, 1} \\
{\left[W /\left(\mathrm{m}^{2} \cdot K\right)\right]}\end{array}$ & $\begin{array}{c}\bar{h}_{c, 2} \\
{\left[W /\left(\mathrm{m}^{2} \cdot K\right)\right]}\end{array}$ \\
\hline $80 \times 800$ & 1.379 & 2.747 & 6.606 & 6.532 & 6.479 & 6.179 \\
$120 \times 1200$ & 1.380 & 2.747 & 6.605 & 6.531 & 6.470 & 6.172 \\
$160 \times 1600$ & 1.381 & 2.748 & 6.604 & 6.530 & 6.467 & 6.170
\end{tabular}

(b) $D=1.71 \mathrm{~cm}, q_{w}=300 \mathrm{~W} / \mathrm{m}^{2}, R a^{*}=1.24 \cdot 10^{4}$

\begin{tabular}{c|c|c|c|c|c|c}
$n_{x} \times n_{z}$ & $\begin{array}{c}\dot{m}_{\text {in }} \\
{[\mathrm{g} /(\mathrm{m} \cdot \mathrm{s})]}\end{array}$ & $\begin{array}{c}\dot{m}_{\text {out }} \\
{[\mathrm{g} /(\mathrm{m} \cdot \mathrm{s})}\end{array}$ & $\begin{array}{c}d_{\psi}(2 \mathrm{H}) \\
{[\mathrm{cm}]}\end{array}$ & $\begin{array}{c}d_{\psi}(3 \mathrm{H} / 2) \\
{[\mathrm{cm}]}\end{array}$ & $\begin{array}{c}\bar{h}_{c, 1} \\
{\left[W /\left(\mathrm{m}^{2} \cdot K\right)\right]}\end{array}$ & $\begin{array}{c}\bar{h}_{c, 2} \\
{\left[W /\left(\mathrm{m}^{2} \cdot K\right)\right]}\end{array}$ \\
\hline $40 \times 800$ & 2.629 & 2.658 & 0.4652 & 0 & 7.292 & 6.751 \\
$80 \times 1600$ & 2.633 & 2.658 & 0.4530 & 0 & 7.294 & 6.750 \\
$160 \times 3200$ & 2.633 & 2.658 & 0.4523 & 0 & 7.294 & 6.750
\end{tabular}

Table 1: Natural convection. Effect of the grid size for (a) $D=7.1 \mathrm{~cm}$, (b) $D=1.71 \mathrm{~cm}\left(q_{w}=300 \mathrm{~W} / \mathrm{m}^{2}\right)$.

\begin{tabular}{l||c|c|c|c}
$(R e, R i)$ & $(200,100)$ & $(200,400)$ & $(400,100)$ & $(400,400)$ \\
\hline$Z_{r}^{*}$, present results & 3.71 & 4.44 & 2.34 & 3.53 \\
\hline$Z_{r}^{*},[16]$ & 3.8 & 4.5 & 2.47 & 3.6 \\
\hline Relative difference & $2.4 \%$ & $1.4 \%$ & $5.3 \%$ & $2.0 \%$
\end{tabular}

Table 2: Dimensionless length of the reversal flow $Z_{r}^{*}$ as a function of the Reynolds number and Richardson number $R i=\operatorname{Ra} /\left(\operatorname{Pr} \operatorname{Re}^{2}\right)$. 


\begin{tabular}{c||cccccccc}
\hline$D[\mathrm{~cm}]$ & 1.02 & 1.23 & 1.40 & 1.71 & 2.02 & 2.30 & 3.80 & 7.10 \\
\hline$R a^{*}$ & 945 & $2.41 \cdot 10^{3}$ & $4.60 \cdot 10^{3}$ & $1.25 \cdot 10^{4}$ & $2.88 \cdot 10^{4}$ & $5.51 \cdot 10^{4}$ & $6.78 \cdot 10^{5}$ & $1.54 \cdot 10^{7}$ \\
$\dot{m}_{\text {in }}[\mathrm{g} /(\mathrm{m} \cdot \mathrm{s})]$ & 1.84 & 2.23 & 2.44 & 2.63 & 2.59 & 2.43 & 1.70 & 1.38 \\
$\dot{m}_{\text {out }}[\mathrm{g} /(\mathrm{m} \cdot \mathrm{s})]$ & 1.84 & 2.23 & 2.44 & 2.66 & 2.75 & 2.75 & 2.71 & 2.75
\end{tabular}

Table 3: Natural convection. Mass flow rate as a function of the wall-spacing $\left(q_{w}=300 \mathrm{~W} / \mathrm{m}^{2}\right)$.

\begin{tabular}{c||cc}
\hline & $R a^{*}=10^{3}$ & $R a^{*}=1.5 \cdot 10^{7}$ \\
\hline$\overline{N u}_{c}=0.82 R a^{*^{0.194}}[1]$ & 3.13 & 20.2 \\
$\overline{N u}_{c, 1}=0.859 R a^{*^{0.183}}$ (present) & 3.04 & 17.7 \\
$\overline{N u}_{c, 2}=0.763 R a^{*^{0.188}}$ (present) & 2.80 & 17.0 \\
\hline$\overline{N u}_{\frac{1}{2}}=0.58 R a^{*^{0.206}}[1]$ & 2.41 & 17.4 \\
$\overline{N u}_{\frac{1}{2}}=0.721 R a^{*^{0.189}}$ present & 2.66 & 16.4 \\
\hline$\theta_{\max }=1.735 R a^{*^{-0.220}}[50]$ & 0.380 & 0.0458 \\
$\theta_{\max }=1.86 R a^{*^{-0.201}}$ (present) & 0.464 & 0.0672
\end{tabular}

Table 4: Natural convection. Comparisons between the present results with previous correlations $[1,50]$ for the range of channel Rayleigh numbers considered (single-plate regime).

\begin{tabular}{c|c||cc}
\hline & & $\begin{array}{c}D=1.02 \mathrm{~cm} \\
\left(R a^{*}=945\right)\end{array}$ & $\begin{array}{c}D=7.1 \mathrm{~cm} \\
\left(R a^{*}=1.54 \cdot 10^{7}\right)\end{array}$ \\
\hline$\varepsilon=0$ & $\bar{T}$ & 332.8 & 338.6 \\
& $T_{\max }$ & 347.7 & 347.7 \\
\hline$\varepsilon=0.1$ & $\bar{T}$ & 331.2 & 334.4 \\
& $T_{\max }$ & 344.9 & 341.4 \\
\hline$\varepsilon=1$ & $\bar{T}$ & 321.2 & 320.7 \\
& $T_{\max }$ & 331.7 & 323.5
\end{tabular}

Table 5: Comparisons between the average and maximum heated wall temperatures $\left(q_{w}=300 \mathrm{~W} / \mathrm{m}^{2}\right)$. 


\begin{tabular}{c|c|c||ccccc}
\hline & & B.C. type & $q_{w}$ & $R a^{*}$ & $\bar{h}_{c v, 1}$ & $\bar{h}_{c v, 2}$ & $T_{\max }$ \\
& & & {$\left[W / m^{2}\right]$} & & {$\left[W /\left(m^{2} \cdot K\right)\right]$} & {$\left[W /\left(m^{2} \cdot K\right)\right]$} & {$[K]$} \\
\hline 1 & $\varepsilon=0$ & $q_{w}$ (N.C.) & 300 & $2.88 \cdot 10^{4}$ & 7.06 & 6.59 & 346.5 \\
\hline \hline 2 & $\varepsilon=0.1$ & $q_{w}$ (Coupled) & 300 & $2.88 \cdot 10^{4}$ & 7.05 & 6.56 & 342.5 \\
$2 a$ & $\varepsilon=0$ & $\bar{q}_{w}^{\prime}$ & 281 & $2.69 \cdot 10^{4}$ & 6.98 & 6.52 & 343.5 \\
$2 b$ & - & $T_{w}(z)$ & $282^{*}$ & $2.71 \cdot 10^{4}$ & 6.63 & 6.60 & 342.7 \\
$2 c$ & - & $\bar{T}_{w}$ & $268^{*}$ & $2.57 \cdot 10^{4}$ & 6.27 & 6.27 & 332.8 \\
\hline \hline 3 & $\varepsilon=1$ & $q_{w}$ (Coupled) & 300 & $2.88 \cdot 10^{4}$ & 6.67 & 6.20 & 327.2 \\
$3 a$ & $\varepsilon=0$ & $\bar{q}_{w}^{\prime}$ & 192 & $1.85 \cdot 10^{4}$ & 6.47 & 6.11 & 329.3 \\
$3 b$ & - & $T_{w}(z)$ & $194^{*}$ & $1.86 \cdot 10^{4}$ & 6.51 & 6.25 & 327.4 \\
$3 c$ & - & $\bar{T}_{w}$ & $186^{*}$ & $1.78 \cdot 10^{4}$ & 5.99 & 5.99 & 321.0
\end{tabular}

Table 6: Comparisons between the different procedures used for the convective part $(D=2.02 \mathrm{~cm})$. N. C.: pure natural convection, Coupled: combined radiation and natural convection, ${ }^{*}$ denotes an output from the temperature distribution, $R a^{*}$ are based on the average wall heat flux given in column 4 . 


\section{FIGURES}

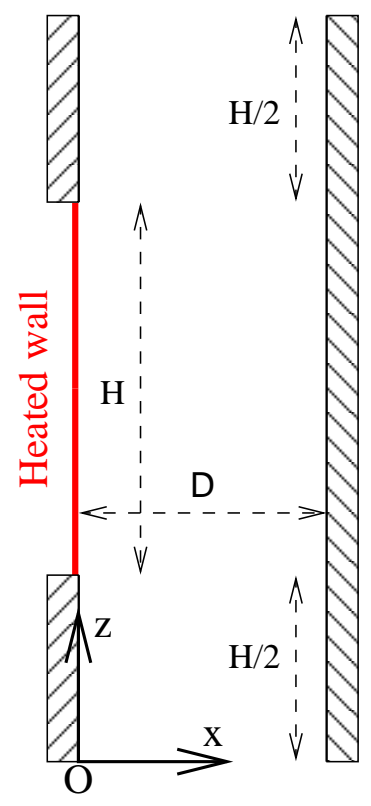

Figure 1: Geometry of the parallel-plate channel. 

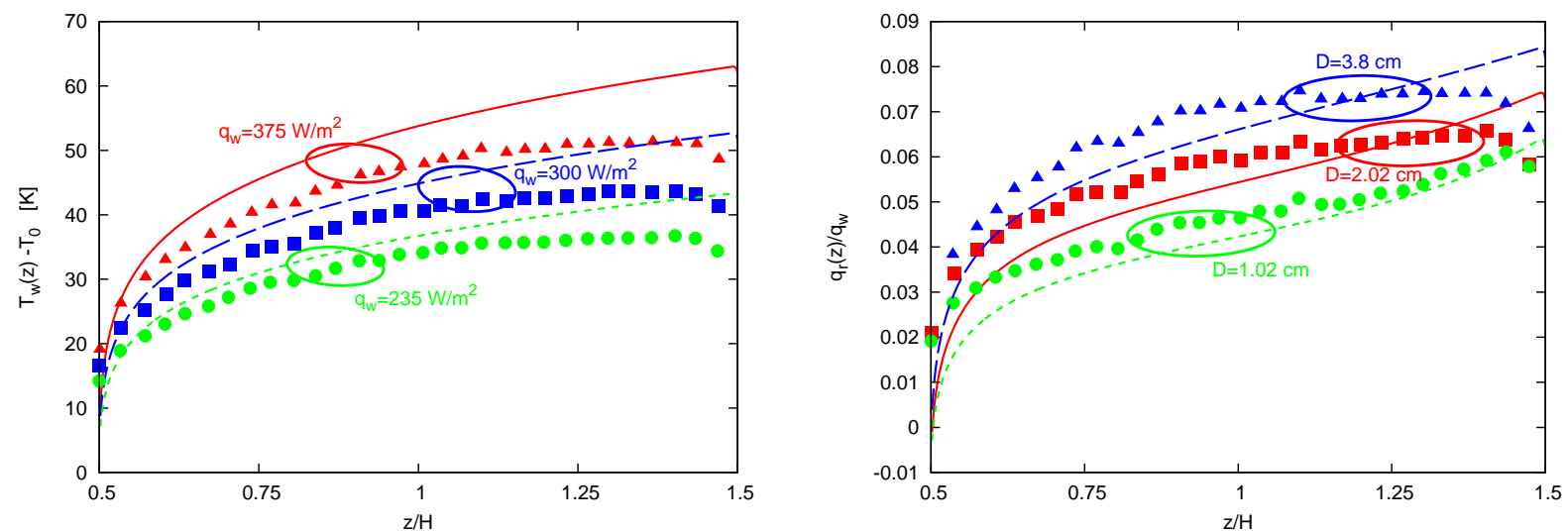

(a) Variations of the heated wall temperature for the (b) Variations of the local radiative flux for three wall three heating rates $(D=2.02 \mathrm{~cm})$. spacings $\left(q_{w}=300 \mathrm{~W} / \mathrm{m}^{2}\right)$.

Figure 2: Variations of (a) the heated wall temperature and (b) the local radiative flux to the imposed wall heat flux versus $z / H(\epsilon=0.1)$. Symbols are experimental data from Webb and Hill [1]. 


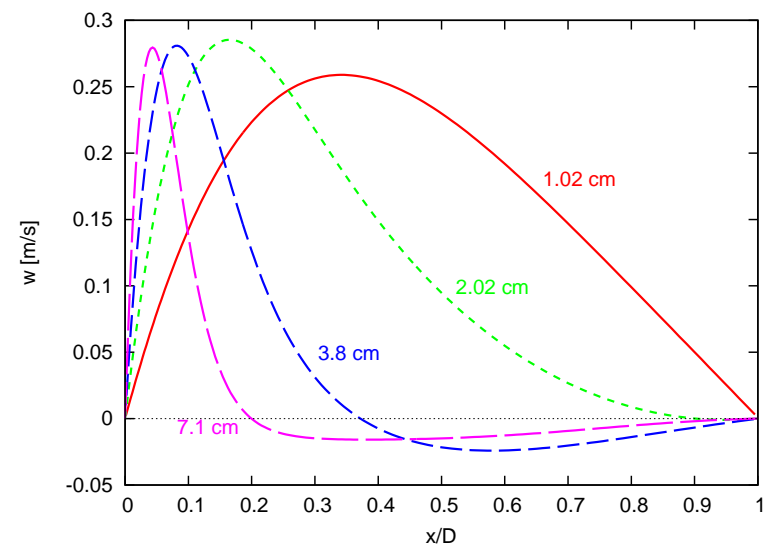

(a) Vertical velocity, $z=3 H / 2$

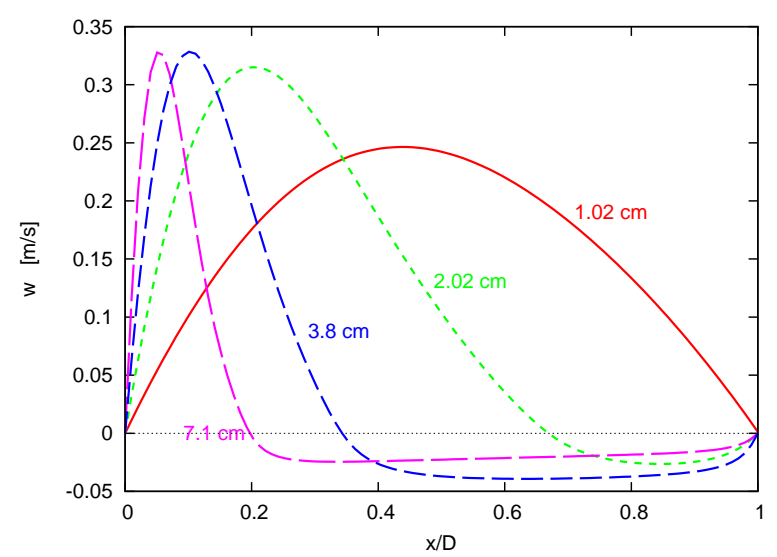

(c) Vertical velocity, $z=2 H$

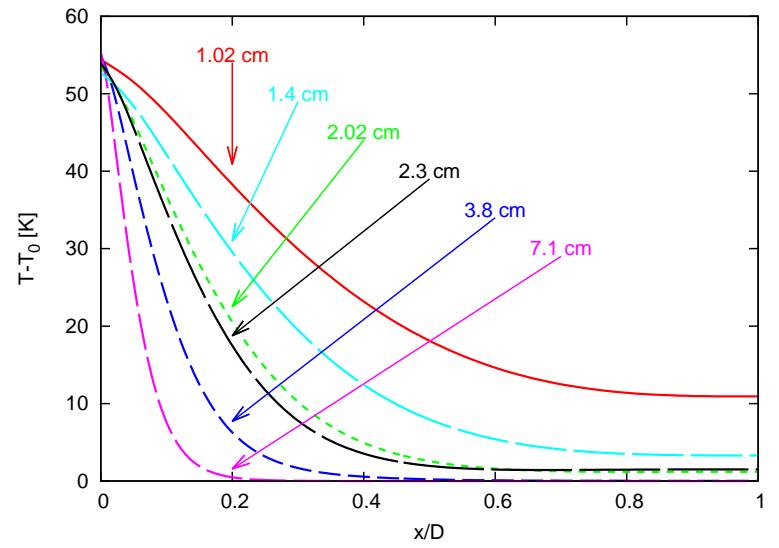

(b) Temperature, $z=3 H / 2$

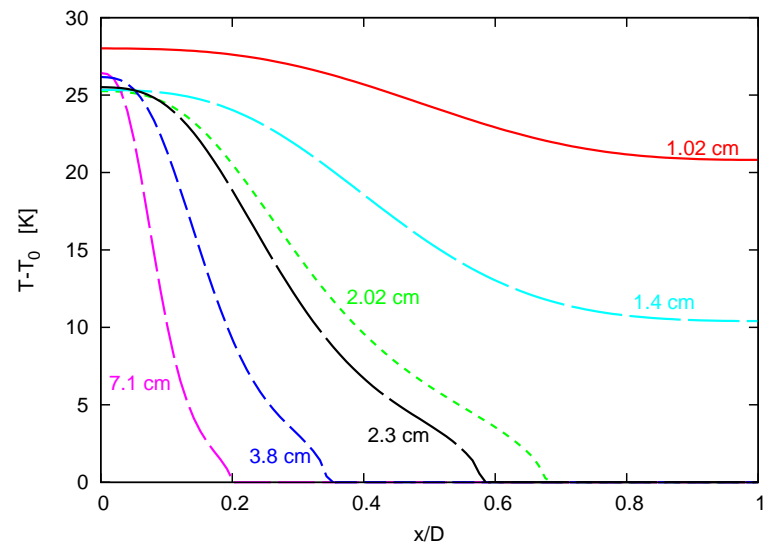

(d) Temperature, $z=2 H$

Figure 3: Natural convection. (a), (c) Vertical velocity profiles and (b), (d) temperature according to the wall spacing $\left(q_{w}=300 \mathrm{~W} / \mathrm{m}^{2}\right)$.

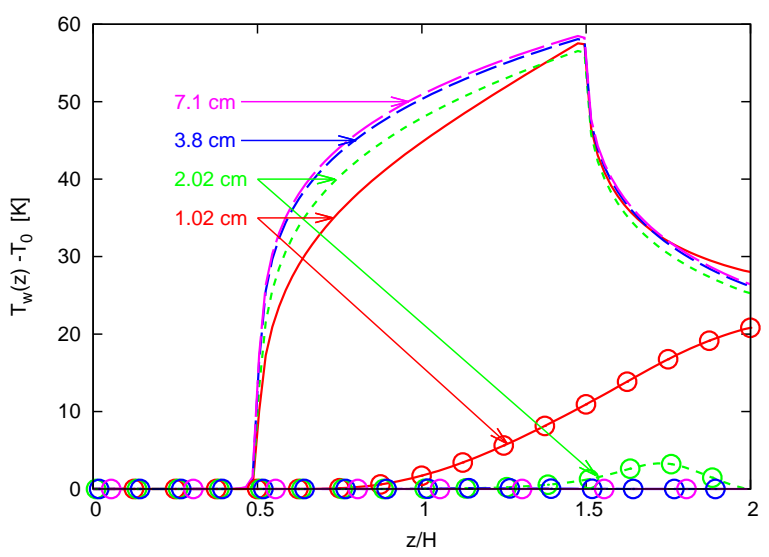

Figure 4: Natural convection. Temperature distribution along the vertical channel walls according to the wall spacing $\left(q_{w}=300 \mathrm{~W} / \mathrm{m}^{2}\right)$. The open circle symbols are for the adiabatic wall. 


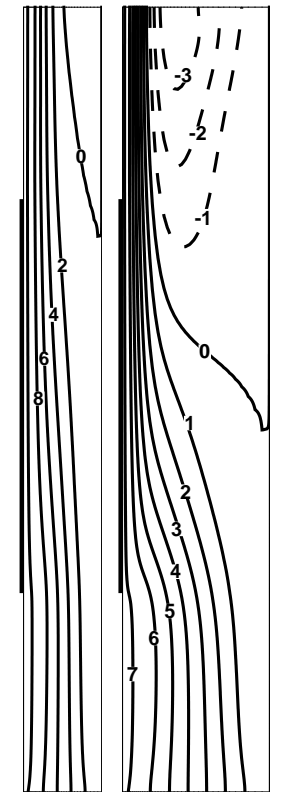

(a) Streamlines

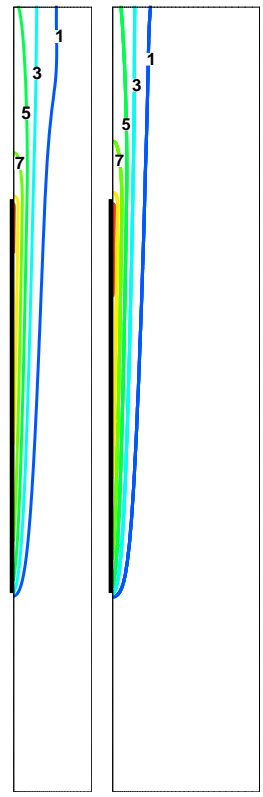

(b) Isotherms

Figure 5: Natural convection. (a) Streamlines $\psi_{i} /[\mathrm{g} /(\mathrm{m} \cdot \mathrm{s})]=0.25 \times i$ and (b) isotherms $T_{i} /[K]=290+5 \times i$ for $D=2.02 \mathrm{~cm}$ and $D=3.8 \mathrm{~cm}\left(q_{w}=300 \mathrm{~W} / \mathrm{m}^{2}\right)$. The horizontal scales are enlarged by $50 \%$.

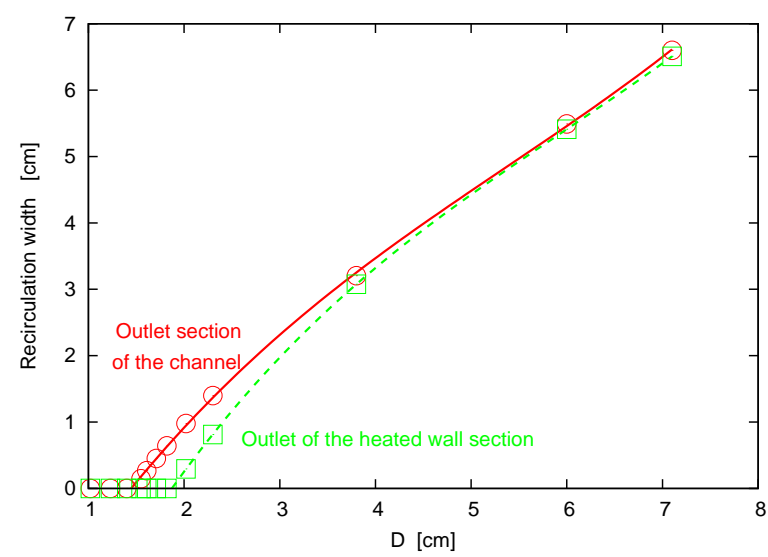

(a) Recirculation widths

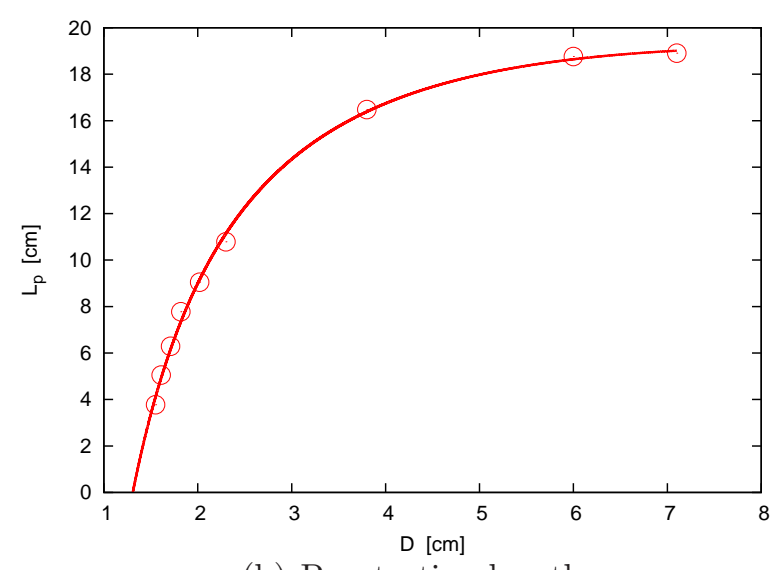

(b) Penetration length

Figure 6: Natural convection. (a) Recirculation widths and (b) penetration length $\left(L_{p}\right)$ versus the channel width $\left(q_{w}=300 \mathrm{~W} / \mathrm{m}^{2}\right)$. 


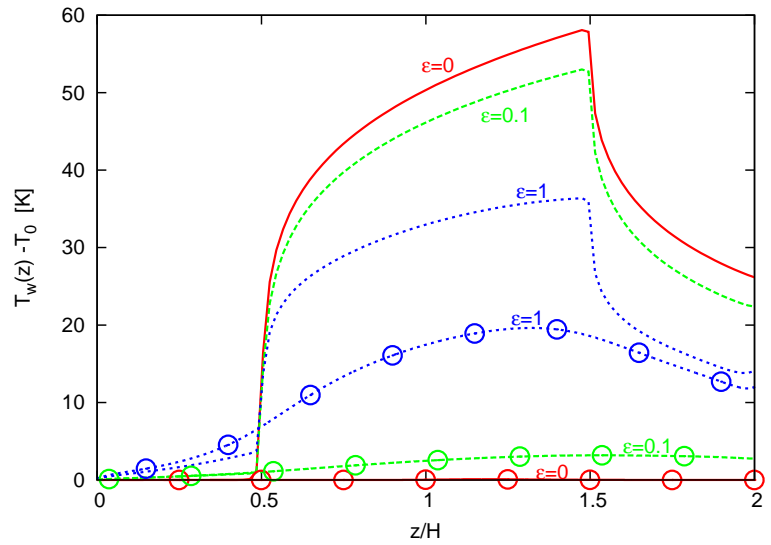

Figure 7: Variation of the heated wall temperature with height according to the emissivity of the vertical walls $\left(D=3.8 \mathrm{~cm}\right.$ and $\left.q_{w}=300 \mathrm{~W} / \mathrm{m}^{2}\right)$. The open circle symbols are for the adiabatic wall. 


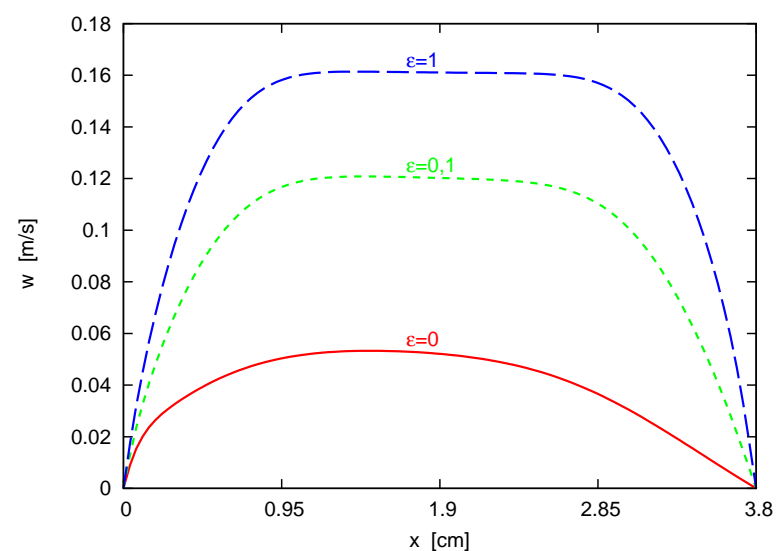

(a) Vertical velocity profile, $z=H / 2$

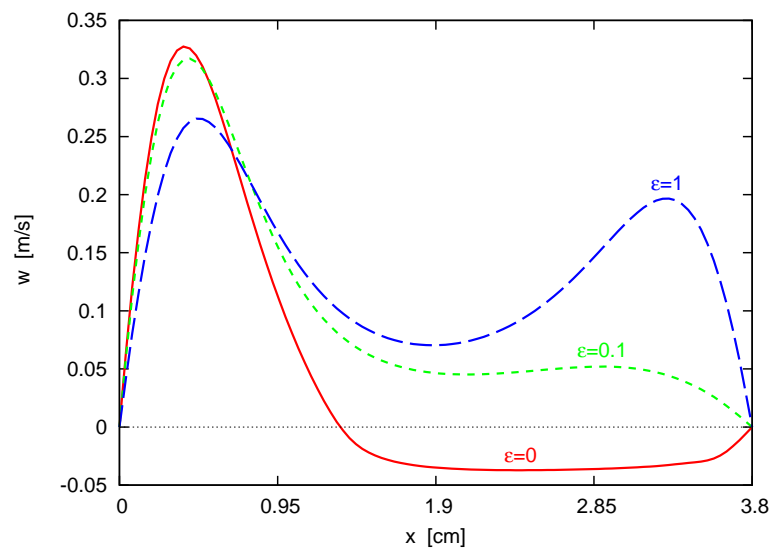

(c) Vertical velocity profile, $z=2 H$

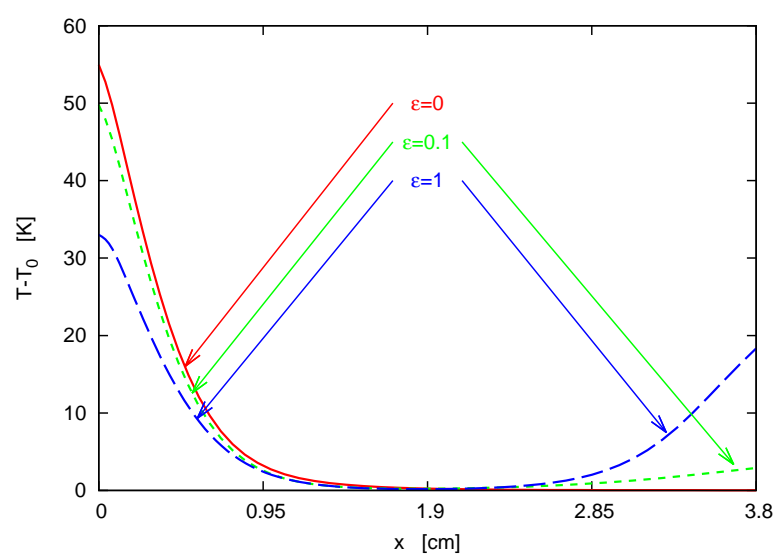

(e) Temperature increase, $z=3 H / 2$

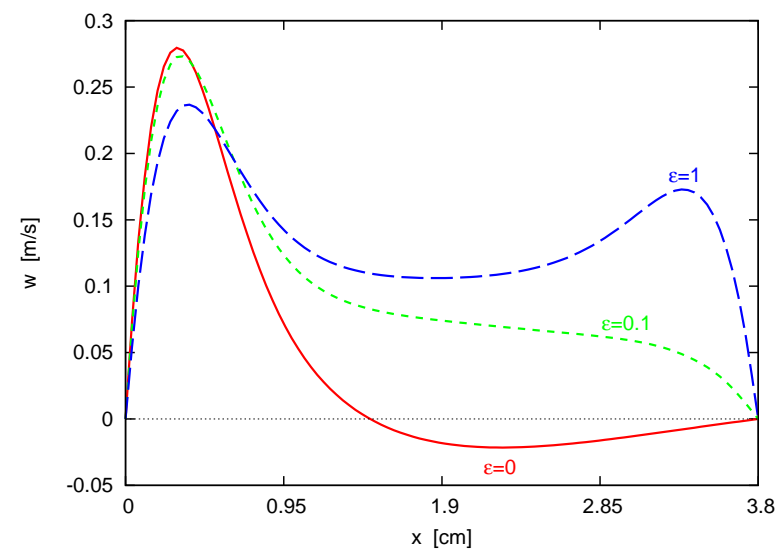

(b) Vertical velocity profile, $z=3 H / 2$

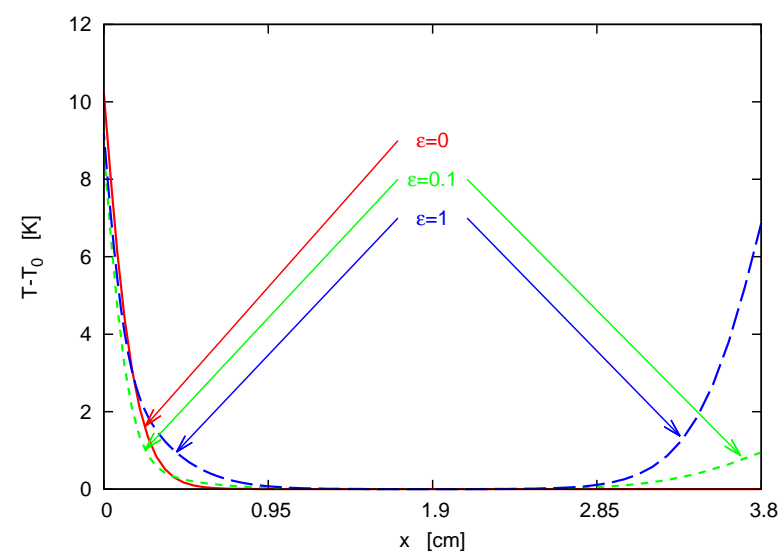

(d) Temperature increase, $z=H / 2$

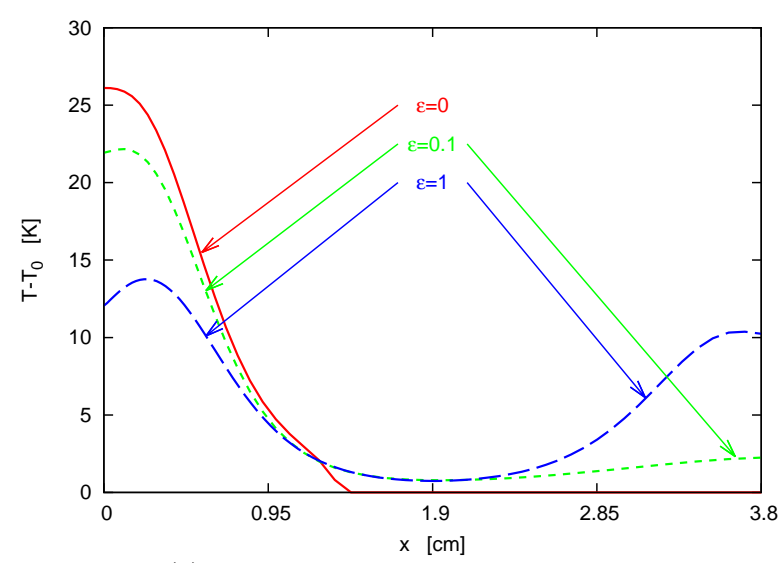

(f) Temperature increase, $z=H$

Figure 8: Vertical velocity profiles (a) at the inlet of the heated wall section $(z=H / 2)$, (b) at the outlet of the heated wall section $(z=3 H / 2)$, (c) at the outlet section of the channel $(z=2 H)$ and temperature gap profiles (d) at the inlet of the heated wall section $(z=H / 2)$, (e) at the outlet of the heated wall section $(z=3 H / 2)$, (f) at the outlet section of the channel $(z=2 H)$ as a function of the emissivity of the vertical walls $\left(D=3.8 \mathrm{~cm}, q_{w}=300 \mathrm{~W} / \mathrm{m}^{2}\right)$. 


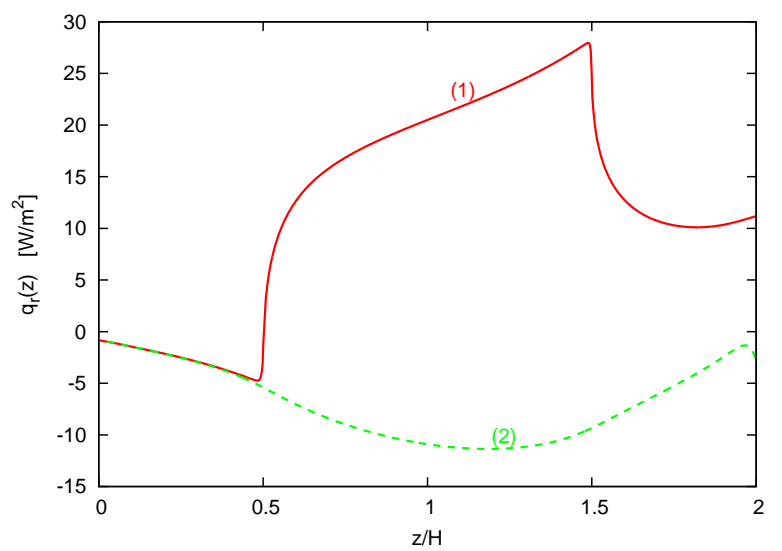

(a) $\epsilon=0.1$

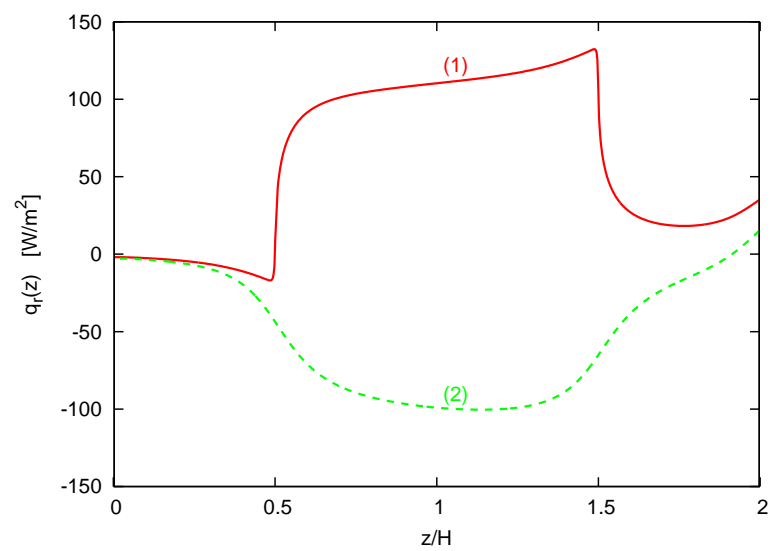

(b) $\epsilon=1$

Figure 9: Distribution of the net radiative heat flux densities along (1) the heated wall and (2) along the adiabatic wall, with (a) $\epsilon=0.1$ and (b) $\epsilon=1\left(D=2.02 \mathrm{~cm}\right.$ and $\left.q_{w}=300 \mathrm{~W} / \mathrm{m}^{2}\right)$.

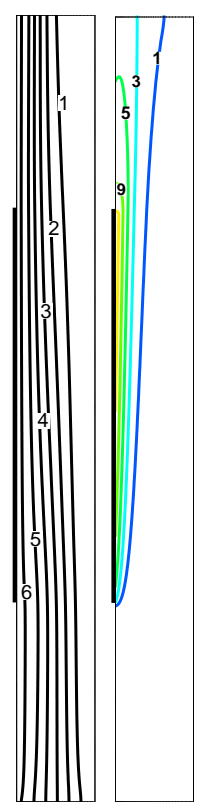

(a) $D=2.02 \mathrm{~cm}, \epsilon=0.1$

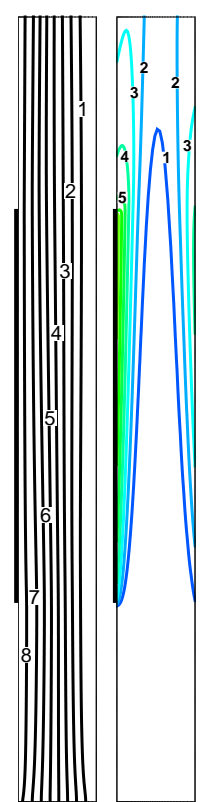

(b) $D=2.02 \mathrm{~cm}, \epsilon=1$

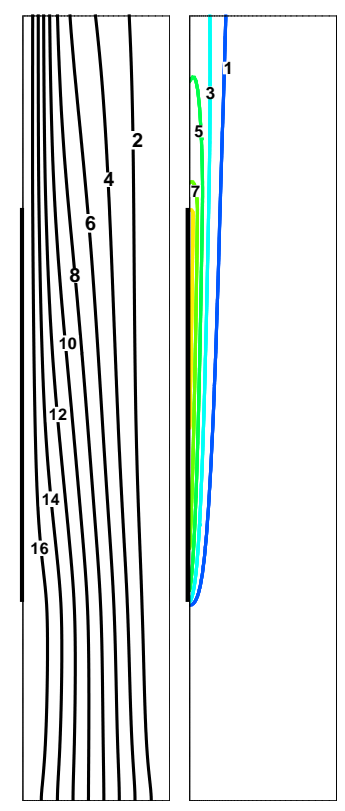

(c) $D=3.8 \mathrm{~cm}, \epsilon=0.1$

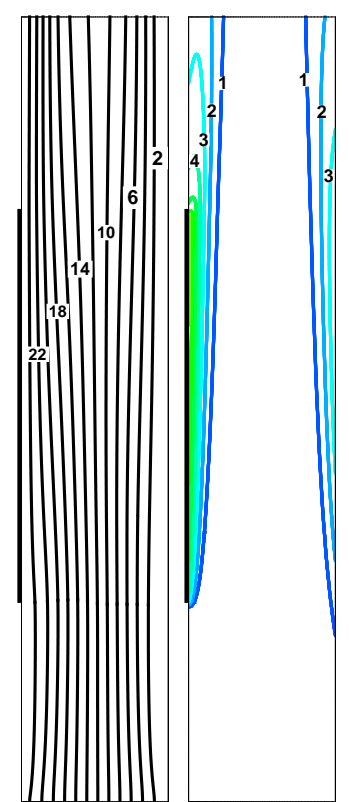

(d) $D=3.8 \mathrm{~cm}, \epsilon=1$

Figure 10: Effect of surface radiation on streamlines $\psi_{i} /[\mathrm{g} /(\mathrm{m} \cdot \mathrm{s})]=0.25 \times i$ (left) and isotherms $T_{i} /[\mathrm{K}]=$ $290+5 \times i$ (right) for (a) $D=2.02 \mathrm{~cm}, \epsilon=0.1$, (b) $D=2.02 \mathrm{~cm}, \epsilon=1$, (c) $D=3.8 \mathrm{~cm}, \epsilon=0.1$, (d) $D=3.8 \mathrm{~cm}, \epsilon=1\left(q_{w}=300 \mathrm{~W} / \mathrm{m}^{2}\right)$. The horizontal scales are enlarged by $50 \%$. 


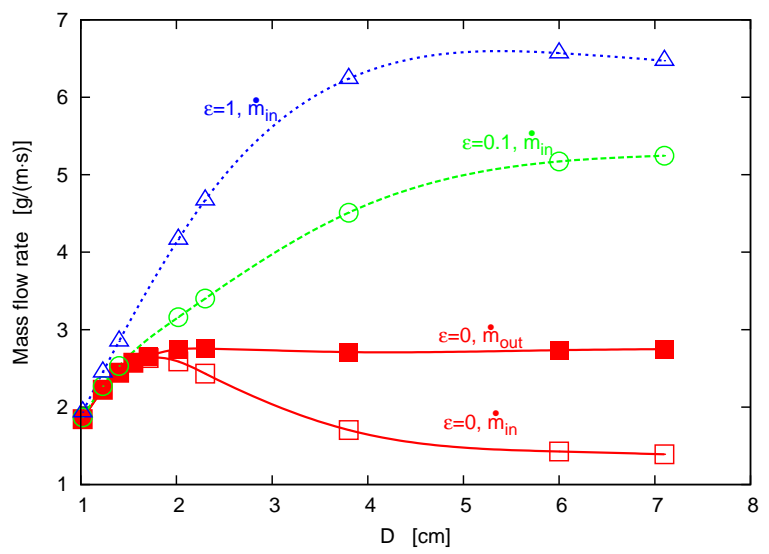

Figure 11: Mass flow rate $\dot{m}_{\text {in }}$ versus the wall spacing for three wall emissivities and $\dot{m}_{\text {out }}$ for $\epsilon=0$. Mass flow rates $\dot{m}_{\text {out }}=\dot{m}_{\text {in }}$ for $\epsilon=0.1$ and $\epsilon=1\left(q_{w}=300 \mathrm{~W} / \mathrm{m}^{2}\right)$.

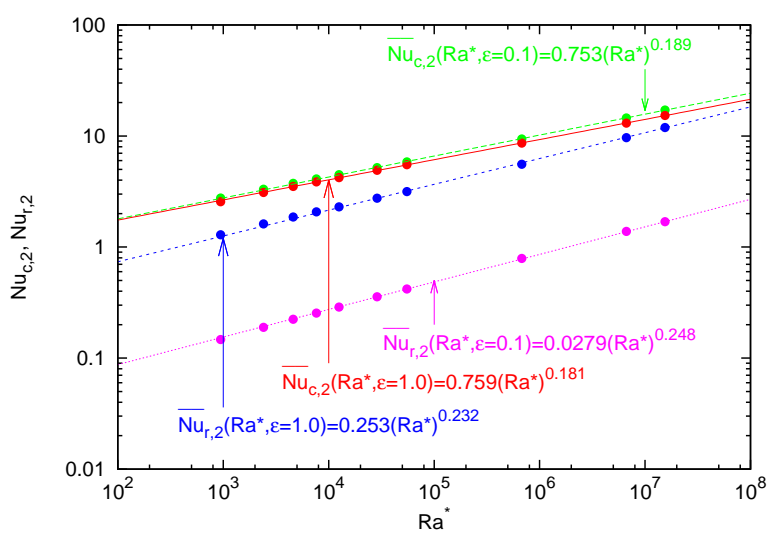

Figure 12: Radiative $\left(\overline{N u}_{r, 2}\right)$ and convective $\left(\overline{N u}_{c, 2}\right)$ Nusselt numbers (eq. 30) versus the channel Rayleigh number for two emissivities of the vertical walls $\left(q_{w}=300 \mathrm{~W} / \mathrm{m}^{2}\right)$. 


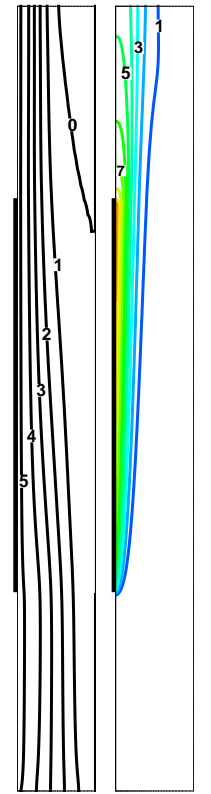

(a) $\bar{q}_{w}^{\prime}=281 \mathrm{~W} / \mathrm{m}^{2}$ and adiabatic surfaces (Tab. 6, row $2 \mathrm{a}$ )

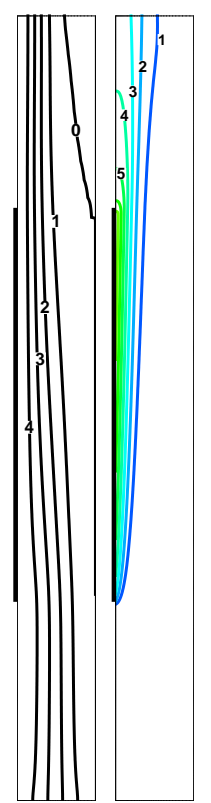

(d) $\bar{q}_{w}^{\prime}=192 \mathrm{~W} / \mathrm{m}^{2}$ and adiabatic surfaces (Tab. 6, row 3a)

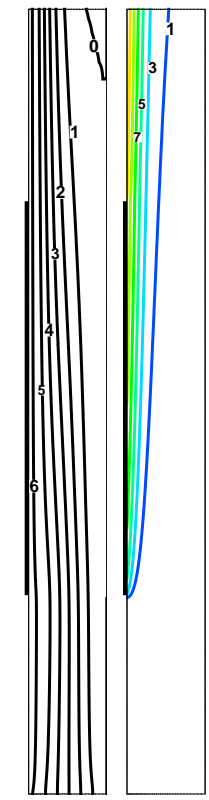

(b) $T_{w}(z)$ and average temperatures on other walls (Tab. 6, row $2 \mathrm{~b})$

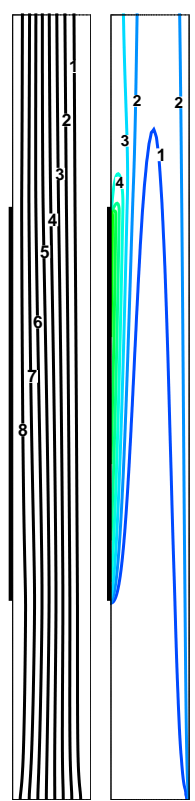

(e) $T_{w}(z)$ and average temperatures on other walls (Tab. 6, row 3b)

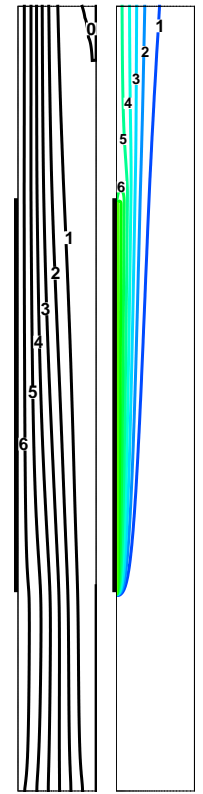

(c) $\bar{T}_{w}=332.8 \mathrm{~K}$ and average temperatures on other walls (Tab. 6, row 2c)

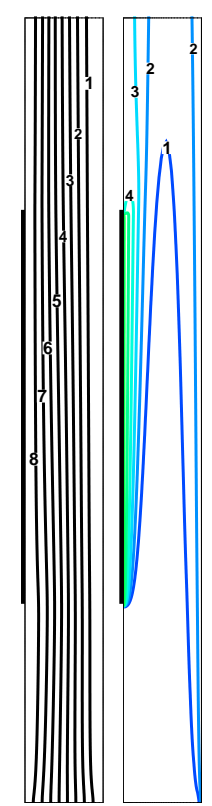

(f) $\bar{T}_{w}=321.0 \mathrm{~K}$ and average temperatures on other walls (Tab. 6, row 3c)

Figure 13: Natural convection. Streamlines $\psi_{i} /[\mathrm{g} /(\mathrm{m} \cdot \mathrm{s})]=0.5 \times i$ (left) and isotherms $T_{i} /[K]=290+5 \times i$ (right) with (a), (d) imposed wall fluxes $\bar{q}_{w}^{\prime}$ and adiabatic surfaces, (b), (e) $T_{w}(z)$ and average temperatures on other walls and (c), (f) imposed average temperatures $\bar{T}_{w}$ and average temperatures on other walls $(D=2.02 \mathrm{~cm})$. The horizontal scales are enlarged by $50 \%$. 


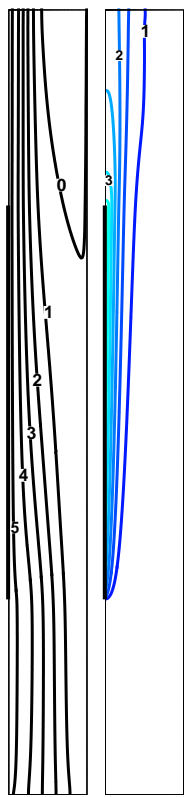

(a) $q_{w}=375 \mathrm{~W} / \mathrm{m}^{2}$, Boussinesq approximation

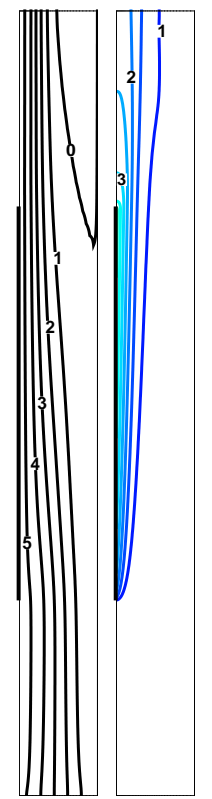

(b) $q_{w}=375 \mathrm{~W} / \mathrm{m}^{2}$, low Mach number approximation

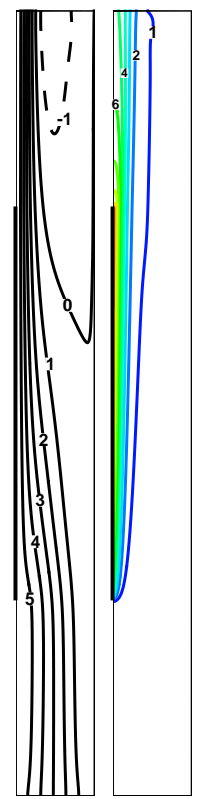

(c) $q_{w}=1400 \mathrm{~W} / \mathrm{m}^{2}$, Boussinesq approximation

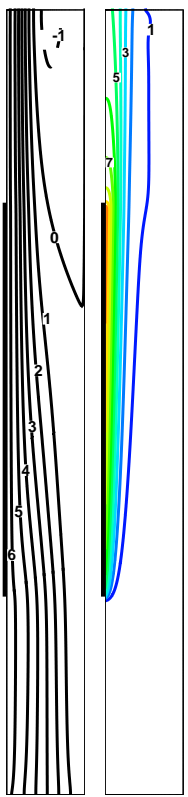

(d) $q_{w}=1400 \mathrm{~W} / \mathrm{m}^{2}$, low Mach number approximation

Figure 14: Natural convection. Streamlines $\psi_{i} /[\mathrm{g} /(\mathrm{m} \cdot \mathrm{s})]=0.5 \times i$ (left) and isotherms $T_{i} /[K]=290+20 \times i$ (right) with (a) $q_{w}=375 \mathrm{~W} / \mathrm{m}^{2}$ and the Boussinesq approximation, (b) $q_{w}=375 \mathrm{~W} / \mathrm{m}^{2}$ and the low Mach number approximation, (c) $q_{w}=1400 \mathrm{~W} / \mathrm{m}^{2}$ and the Boussinesq approximation, (d) $q_{w}=1400 \mathrm{~W} / \mathrm{m}^{2}$ and the low Mach number approximation $(D=2.02 \mathrm{~cm})$. The horizontal scales are enlarged by $50 \%$. 